\title{
Dispersive Readout of Majorana Qubits
}

\author{
Thomas B. Smith $\odot,{ }^{1}$ Maja C. Cassidy $\odot,{ }^{2}$ David J. Reilly, ${ }^{1,2}$ Stephen D. Bartlett $\odot,{ }^{1}$ and \\ Arne L. Grimsmo ${ }^{1, *}$ \\ ${ }^{1}$ ARC Centre of Excellence for Engineered Quantum Systems, School of Physics, The University of Sydney, \\ Sydney, New South Wales 2006, Australia \\ ${ }^{2}$ Microsoft Quantum Sydney, The University of Sydney, Sydney, New South Wales 2006, Australia
}

(Received 7 September 2020; accepted 20 October 2020; published 10 November 2020)

\begin{abstract}
We analyze a readout scheme for Majorana qubits based on dispersive coupling to a resonator. We consider two variants of Majorana qubits: the Majorana transmon and the Majorana box qubit. In both cases, the qubit-resonator interaction can produce sizeable dispersive shifts in the megahertz range for reasonable system parameters, allowing for submicrosecond readout with high fidelity. For Majorana transmons, the light-matter interaction used for readout manifestly conserves Majorana parity, which leads to a notion of quantum nondemolition (QND) readout that is stronger than for conventional charge qubits. In contrast, Majorana box qubits only recover an approximately QND readout mechanism in the dispersive limit where the resonator detuning is large. We also compare dispersive readout to longitudinal readout for the Majorana box qubit. We show that the latter gives faster and higher fidelity readout for reasonable parameters, while having the additional advantage of being manifestly QND, and so may prove to be a better readout mechanism for these systems.
\end{abstract}

DOI: 10.1103/PRXQuantum.1.020313

\section{INTRODUCTION}

Topological phases of matter offer a promising platform for quantum information processing, as qubits encoded into the degenerate ground states of these exotic phases should be extremely robust to errors [1-3]. Engineered symmetry-protected topological phases have been proposed and recently investigated in one-dimensional hybrid semiconductor-superconductor nanowires [4-18]. Under specific conditions, these nanowires may enter a topological superconducting phase, distinguished by the emergence of Majorana zero-energy modes (MZMs). Fabricated devices with multiple nanowires could allow quantum computation with these MZMs [19-26].

Majorana-based quantum computing requires a measurement scheme for MZMs [27-35], which ideally should be fast and have high fidelity. In measurementonly approaches to topological quantum computation, such measurements take on an especially important role, replacing braiding for the implementation of topologically protected quantum logic gates $[25,26,36,37]$. Here,

*arne.grimsmo@gmail.com

Published by the American Physical Society under the terms of the Creative Commons Attribution 4.0 International license. Further distribution of this work must maintain attribution to the author(s) and the published article's title, journal citation, and DOI. measurements must in addition be quantum nondemolition (QND). The QND property ensures that the measured observable is a conserved quantity, and constrains the postmeasurement state to be an eigenstate of the observable, such that repeated measurements give the same outcome. This is a critical requirement for measurement-only topological quantum computation with MZMs, where the measurements determine the dynamics of the system. Beyond the implementation of topologically protected logic gates, measurements also play a key role in other aspects of Majorana-based quantum computing such as magic state distillation [38].

Looking broadly at measurement schemes for solidstate quantum computing, a standard approach has been to couple a qubit-state-dependent charge dipole to the electric field of a resonator, which is used as a measurement probe [39]. A QND readout scheme for Majorana qubits based on parametric modulation of a qubit-resonator coupling was recently introduced in Ref. [30]. However, the workhorse of measurement schemes for solid-state qubits is dispersive readout, which has been very successful for superconducting [40,41], semiconducting [42-44], and hybrid semiconductor-superconductor qubits $[45,46]$. In these schemes the resonator is tuned far off-resonance from the qubit frequency, and acquires a qubit-state-dependent frequency shift. It is natural to ask whether such a dispersive readout scheme can offer similar advantages for Majorana qubits, and to what extent it can satisfy the stringent 
QND requirements that are demanded by measurementonly topological quantum computation.

In this paper, we investigate a dispersive readout scheme for two prototypical Majorana qubits: the Majorana transmon [24,47-49] and the Majorana box qubit [25,26]. These two designs are distinguished by whether the two topological wire segments that host the four MZMs form two distinct superconducting charge islands or a single island with a uniform superconducting phase, respectively. We calculate the qubit-state-dependent dispersive shift that arises when these Majorana qubits are capacitively coupled to the electric field of a readout resonator. The size of these dispersive shifts directly determine the rate at which one can perform qubit readout by driving the resonator and observing the phase shift of the reflected field [50]. It also therefore determines the clock frequency in measurement-only approaches to quantum computation with MZMs.

Majorana qubits differ from conventional superconducting charge qubits, such as the Cooper pair box and the transmon [51], as a dispersive shift for a Majorana qubit can arise despite the fact that the interaction with the resonator does not induce (virtual) transitions between the two logical qubit states. Instead, the shifts result from (virtual) transitions to excited states outside the qubit subspace. This is especially beneficial for the Majorana transmon, where dispersive shifts arise through a qubitresonator interaction that conserves the Majorana charge parity. For the Majorana box qubit, the Majorana parity is only approximately conserved in a limit of large frequency detuning from the resonator.

For both Majorana transmons and Majorana box qubits, we find that dispersive shifts can be comparable to those of conventional transmon qubits [41] and nanowire quantum dots [44]. Specifically, for reasonable system parameters, we predict dispersive shifts in the megahertz range. Measuring the qubit necessarily requires lifting the MZM degeneracy, and the corresponding qubit frequency is in the range 1-2 GHz. Our results suggest that submicrosecond high-fidelity QND readout is feasible for Majorana qubits.

The remainder of the paper is organized as follows. We give a high-level introduction to dispersive coupling between a Majorana qubit and a resonator in Sec. II. In Secs. III and IV we describe in detail the dispersive coupling for a Majorana transmon and a Majorana box qubit, respectively. For both qubit variants, we calculate the dispersive frequency shift of a readout resonator from secondorder Schrieffer-Wolff perturbation theory for a range of system parameters using numerical diagonalization. We also provide simple, approximate analytical expressions. We estimate the resulting measurement timescales and fidelities (in the absence of any unwanted qubit decoherence or noise in the system parameters; see, e.g., [34,35, $52,53])$ in Sec. V. We compare the results for dispersive readout to the longitudinal readout scheme introduced in Ref. [30]. Finally, in Sec. VI we discuss the implications of our results for dispersive readout of Majorana qubits.

\section{LIGHT-MATTER INTERACTION FOR MAJORANA QUBITS}

We begin by presenting a high-level overview of the interaction between a Majorana qubit and an electromagnetic resonator, focusing on the dispersive coupling regime. Such a coupling provides the underlying physical mechanism that can be used for dispersive qubit readout [39].

The resonator-based readout schemes considered in this paper involve capacitive coupling of the charge degree of freedom of the measured system (the qubit) to the electric field of a nearby resonator. That is, we have an interaction Hamiltonian of the form

$$
\hat{H}_{\text {int }}=\hat{Q} \hat{V}_{r}=i \hbar \lambda \hat{N}\left(\hat{a}^{\dagger}-\hat{a}\right),
$$

where $\hat{Q}=e \hat{N}$ is a charge operator for the qubit system, $\hat{V}_{r} \sim i\left(\hat{a}^{\dagger}-\hat{a}\right)$ is the voltage bias on the qubit due to the resonator and $\lambda$ quantifies the interaction strength. For simplicity, we model the resonator by a single harmonic oscillator mode with annihilation (creation) operator $\hat{a}$ $\left(\hat{a}^{\dagger}\right)$.

The physics resulting from the coupling described by Eq. (1) depends on the internal level structure of the qubit system. Given that the charge number operator $\hat{N}$ can have off-diagonal matrix elements in the qubit eigenbasis, the absorption or emission of a resonator photon can induce a transition between eigenstates in the qubit system. The internal level structure of the qubit can lead to selection rules where only certain transitions are allowed. We show below that different Majorana qubit designs give rise to different selection rules, and discuss the consequences of this for QND readout.

We consider two distinct types of Majorana qubits, illustrated in Fig. 1. Each topological superconductor hosts a pair of Majorana edge modes, and we associate to each edge mode a Hermitian Majorana fermion operator $\hat{\gamma}_{i}$, with anticommutation relations $\left\{\hat{\gamma}_{i}, \hat{\gamma}_{j}\right\}=2 \delta_{i j}$. Because of charge conservation, a minimum of two topological superconductors are required to encode a Majorana qubit, for a total of four MZMs. We identify two broad classes of Majorana qubits, depending on whether these topological superconductors form one or two distinct superconducting charge islands. The Majorana transmon qubit is representative of a configuration where the two topological superconductors form two distinct islands, and the relevant charge degree of freedom for readout is the difference in charge between these two islands. Variations of this configuration can include grounding one of the two superconducting islands, and/or introducing a Josephson tunnel 


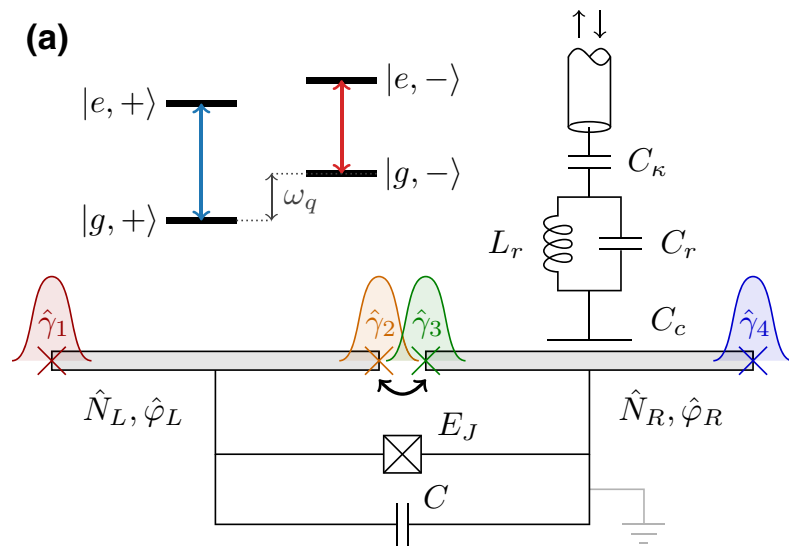

Majorana transmon

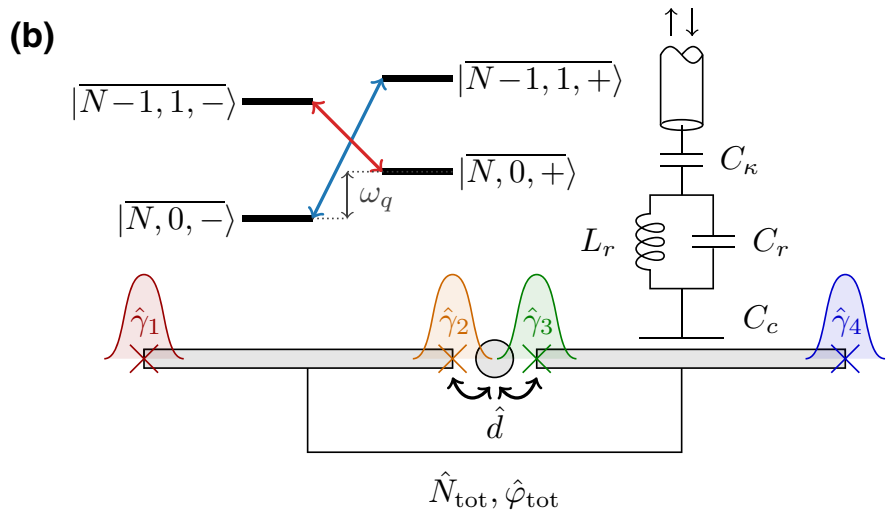

Majorana box qubit

FIG. 1. Illustration of readout for two Majorana qubit variants. Topological superconductors (gray rectangles) host MZMs $\hat{\gamma}_{i}$, and qubit readout corresponds to a measurement of $i \hat{\gamma}_{2} \hat{\gamma}_{3}$. Majorana wavefunctions can be made to overlap either by (a) direct tunneling or (b) tunneling to a proximal quantum dot (gray circle). The resonator (illustrated by an $L C$ oscillator) is capacitively coupled to the island charge. Alternative resonator-island coupling geometries are possible, including coupling to the quantum dot in (b). (a) Majorana transmon qubit: the topological superconductors form two distinct superconducting islands, shunted by a Josephson junction with energy $E_{J}$ (one of the islands may be grounded). The level diagram illustrates low-energy eigenstates labeled $|g, \pm\rangle$ and $|e, \pm\rangle$. The blue and red arrows indicate allowed transitions induced by the qubit-resonator interaction. These allowed transitions conserve Majorana parity. (b) Majorana box qubit: the topological superconductors are shunted by a trivial superconductor, and the device forms a single superconducting island. The level diagram labels dressed eigenstates of the coupled superconducting island-dot system. In this case, the resonator induces transitions between dressed states of different dot occupancy and Majorana parity.

coupling between the islands and ground [24]. However, it should be noted that connecting one of the islands to ground in this manner could increase the rate of quasiparticle poisoning events [53]. For the Majorana box qubit (also referred to as the Majorana loop qubit), the two topological superconductors are shunted by a trivial superconductor to form a single superconducting island. In this case, a charge dipole can be formed by tunnel coupling to a proximal quantum dot, providing a mechanism for readout.

The physics of these devices is described in more detail in the following sections, and we here only give a high-level discussion of their internal level structure and selection rules. For the Majorana transmon in Fig. 1(a), energy levels can be labeled $|g, \pm\rangle,|e, \pm\rangle, \ldots$, where $g, e, \ldots$ denote a transmonlike ladder of eigenstates, and \pm denotes the eigenvalue of $i \hat{\gamma}_{2} \hat{\gamma}_{3}= \pm 1$, the Majorana parity we wish to measure. As indicated in the level diagram in Fig. 1(a), only transitions that conserve the Majorana parity are allowed. This means that the Majorana parity is conserved during readout and that the interaction is manifestly QND with respect to this quantity. This stronger-than-usual form of QND measurement stems from the fractional and nonlocal nature of the MZMs [25], and was dubbed the topological QND (TQND) measurement in Ref. [30]. This TQND property plays a key role in maintaining the topological protection of measurementbased approaches to implementing quantum logic gates.

For the Majorana box qubit, MZMs are tunnel coupled to a proximal quantum dot, as illustrated in Fig. 1(b).
The dot might be formed naturally between two topological superconductors due to the boundary conditions set by the superconducting-semiconducting interface [54]. In our analysis we assume that this quantum dot has wellseparated energy levels, and for simplicity only a single level that is energetically accessible.

Charge tunneling between the topological superconducting island and the dot provides a mechanism for readout. Because the superconducting island charge is no longer conserved, the eigenstates of the qubit-dot system are dressed states where the Majorana edge modes are partially localized on the dot. These dressed states are illustrated in the level diagram in Fig. 1(b). In a readout protocol, the tunnel coupling should be turned on gradually, such that the system evolves adiabatically from the bare to the dressed eigenstates, and we label the dressed eigenstates by the states they are adiabatically connected to in the absence of tunneling. Qubit readout corresponds to distinguishing the dressed states adiabatically connected to the degenerate qubit ground space.

As indicated in the level diagram in Fig. 1(b), the relevant transitions for coupling to the resonator involve a single charge transfer from the island to the dot, or vice versa. This charge transfer also flips the (dressed) Majorana parity. In this case, an approximately QND interaction can still be achieved in the dispersive regime, where the resonator is far detuned from any internal transition, such that the resonator-induced transitions indicated in Fig. 1(b) are purely virtual. However, it is a notable difference 
between the Majorana transmon and the Majorana box qubit readout scheme that the former has the advantage of a manifestly QND interaction independent of whether the system is in the dispersive regime or not.

It is worth noting that the joint parity of the Majorana box qubit and the dot is, in fact, preserved by the transitions indicated in Fig. 1(b). One can therefore perform a QND measurement of this joint parity, and in principle infer the Majorana parity, given that the state of the dot can be determined with certainty before and after the measurement $[32,33]$.

As an aside, the lack on an exact QND interaction for the dispersive readout of the Majorana box qubit can be contrasted to the longitudinal readout scheme proposed in Ref. [30], where modulation of a system parameter is used to activate a parity conserving qubit-resonator coupling. This coupling arises independently of the frequency detuning of the resonator from any internal qubit transition. Therefore, this longitudinal readout scheme can be used in a regime where the detuning is large enough that any Majorana parity nonconserving terms are negligible, yet still achieving a large readout signal-to-noise ratio. We return to a brief comparison with Ref. [30] in Sec. V and Appendix E.

For the purpose of qubit readout, real transitions between qubit-system eigenstates are undesirable. An effective interaction suitable for readout is recovered from Eq. (1) in the dispersive regime. This refers to a coupling regime where the resonator frequency is far off-resonance from any relevant transitions between qubit states that are allowed by the selection rules. The transitions to higher energy levels indicated in Figs. 1(a) and 1(b) are then only virtual transitions. In this situation, Eq. (1) can be treated perturbatively, leading to an effective interaction of the form (for both types of Majorana qubit)

$$
\hat{H}_{\text {disp }}=\hbar \omega_{r} \hat{a}^{\dagger} \hat{a}+\frac{\hbar \omega_{q}}{2} \hat{\sigma}_{z}+\hbar \chi_{q} \hat{\sigma}_{z} \hat{a}^{\dagger} \hat{a} .
$$

Here $\omega_{r}$ is the resonator frequency, $\hbar \omega_{q}$ is the energy splitting between the two eigenstates used to encode a qubit, and $\hat{\sigma}_{z}$ is the corresponding logical Pauli- $Z$ operator. In general, the $\omega_{r, q}$ include Lamb shifts due to the qubit-resonator coupling. Finally, $\chi_{q}$ is the qubit-statedependent dispersive frequency shift of the resonator. Under this Hamiltonian, the qubit states can be distinguished by detecting a phase shift of the resonator under a coherent drive at the resonator frequency [39,40,50,51]. The speed of such a measurement is set by the magnitude of the dispersive shift $\chi_{q}$. We give a derivation of Eq. (2) starting from Eq. (1), for a generic multilevel system, in Appendix A. Throughout this paper, we compute $\chi_{q}$ for three different qubit types labeled $q \in\{t, m t, m b\}$, for a conventional transmon, a Majorana transmon, and a Majorana box qubit, respectively.
It is important to emphasize that although Eq. (2) is QND with respect to the logical $\hat{\sigma}_{z}$ operator, this Hamiltonian is an approximation to the underlying light-matter interaction, Eq. (1). The TQND property of the Majorana transmon refers to the fact that parity protection is manifest at the more fundamental level of Eq. (1). As discussed briefly above, and in more detail in the following, dispersive readout for the Majorana box qubit is not TQND in the same strong sense as for the Majorana transmon. Both the Majorana transmon and the Majorana box qubit, however, share the feature that no transitions are allowed between the two lowest energy eigenstates used to form a qubit. Instead, (virtual) transitions out of the qubit subspace are used to realize a readout mechanism. This is in contrast to conventional superconducting charge qubits, such as the transmon and the Cooper pair box, where the light-matter interaction causes transitions between the energy eigenstates that define the qubit [51]. In this case, the readout mechanism introduces a source of error in the form of Purcell decay, wherein the qubit may relax via emission of a photon via the resonator [55]. (We note that we have restricted our notion of measurement back action to the readout mechanism itself. Additional unwanted effects that may be introduced such as quasiparticle poisoning [53] or heating are not treated here.)

In the following sections, we describe the dispersive readout schemes for the Majorana transmon and the Majorana box qubit in detail.

\section{MAJORANA TRANSMON QUBIT}

\section{A. Model for the qubit}

A Majorana transmon qubit, shown in Fig. 1(a), consists of two distinct charge islands that are shunted by a Josephson junction. Each island, labeled $\alpha \in\{L, R\}$, is in a topological superconducting phase and has electron number operator $\hat{N}_{L, R}$ and dimensionless superconducting phase operator $\hat{\varphi}_{L, R}$, satisfying $\left[\hat{N}_{\alpha}, e^{i \hat{\varphi}_{\beta} / 2}\right]=\delta_{\alpha \beta} e^{i \hat{\varphi}_{\beta} / 2}$ with $\alpha, \beta \in\{L, R\}$. The charging energy and conventional Cooper pair tunneling between the two islands is captured by a Hamiltonian

$$
\hat{H}_{T}=E_{C}\left(\hat{N}-n_{g}\right)^{2}-E_{J} \cos \hat{\varphi},
$$

where $\hat{N} \equiv\left(\hat{N}_{L}-\hat{N}_{R}\right) / 2$ and $\hat{\varphi} \equiv \hat{\varphi}_{L}-\hat{\varphi}_{R}, E_{C}$ is the charging energy due to capacitive coupling of the two islands $n_{g}$ represents an offset charge, and $E_{J}$ is the Josephson coupling due to Cooper pair tunneling across the Josephson junction. The transmon regime is characterized by $E_{J} \gg E_{C}$ [51]. Note that here we use a convention where $\hat{N}$ counts the number of electrons rather than the number of Cooper pairs, such that we have the following action on charge eigenstates:

$$
\hat{N}|N\rangle=N|N\rangle,
$$




$$
e^{ \pm i \hat{\varphi}}|N\rangle=|N \pm 2\rangle
$$

We have neglected the capacitances of each superconducting island to ground, and assume the long-island limit where MZMs located on the same island are well separated.

Variations of the Majorana transmon include grounding one of the two islands (such that we can set, e.g., $\hat{\varphi}_{L}=0$ and $\hat{\varphi}=\hat{\varphi}_{R}$ ) and/or introducing a Josephson coupling to a bulk superconductor in addition to the Josephson coupling between the two islands [24]. These variations are qualitatively similar, and our results extend to these cases without any significant modification.

To read out this qubit, the MZMs corresponding to $\hat{\gamma}_{2}$ and $\hat{\gamma}_{3}$ are brought together and the combined parity $i \hat{\gamma}_{2} \hat{\gamma}_{3}$ is measured. When these two MZMs are brought together (see Fig. 1), their interaction is governed by a tunneling Hamiltonian $[4,24,47,49]$

$$
\hat{H}_{M}=-E_{M} i \hat{\gamma}_{2} \hat{\gamma}_{3} \cos \left(\frac{\hat{\varphi}+\varphi_{x}}{2}\right)
$$

where $E_{M}$ is proportional to the wavefunction overlap of the MZMs. This expression accounts for the fact that the qubit loop might enclose an external flux $\Phi_{x}$, where in we have defined $\varphi_{x}=2 \pi \Phi_{x} / \Phi_{0}$ with $\Phi_{0}=h / 2 e$ the magnetic flux quantum. In Appendix B we compare the direct tunneling model Eq. (5) with a model where the two islands are coupled to a common quantum dot, acting as a mediator. The main outcome of this comparison is that, when the energy penalty to occupy the quantum dot becomes large, the two models are equivalent.

The full Majorana transmon qubit Hamiltonian is

$$
\hat{H}_{\mathrm{MT}}=\hat{H}_{T}+\hat{H}_{M}
$$

Since $i \hat{\gamma}_{2} \hat{\gamma}_{3}$ commutes with $\hat{H}_{\mathrm{MT}}$, the eigenstates can conveniently be labeled by two quantum numbers $|j, a\rangle$, where $j=g, e, f, \ldots$ denote a transmonlike ladder of eigenstates and $a= \pm$ denotes the eigenvalue of $i \hat{\gamma}_{2} \hat{\gamma}_{3}=$ \pm 1 . The level structure is shown in Fig. 2 for (a) $E_{M}=0$ and (b) $E_{M}>0$.

A simplified Hamiltonian can be found by following the standard approach of treating the transmon degree of freedom as a Kerr nonlinear oscillator [39,51]. To keep the discussion simple, we set the offset charge and external flux to zero, $n_{g}=0, \varphi_{x}=0$, for the remainder of this section. We can introduce ladder operators via

$$
\begin{aligned}
& \hat{N}=i\left(\frac{E_{J}}{2 E_{C}}\right)^{1 / 4}\left(\hat{b}^{\dagger}-\hat{b}\right), \\
& \hat{\varphi}=\left(\frac{2 E_{C}}{E_{J}}\right)^{1 / 4}\left(\hat{b}^{\dagger}+\hat{b}\right),
\end{aligned}
$$
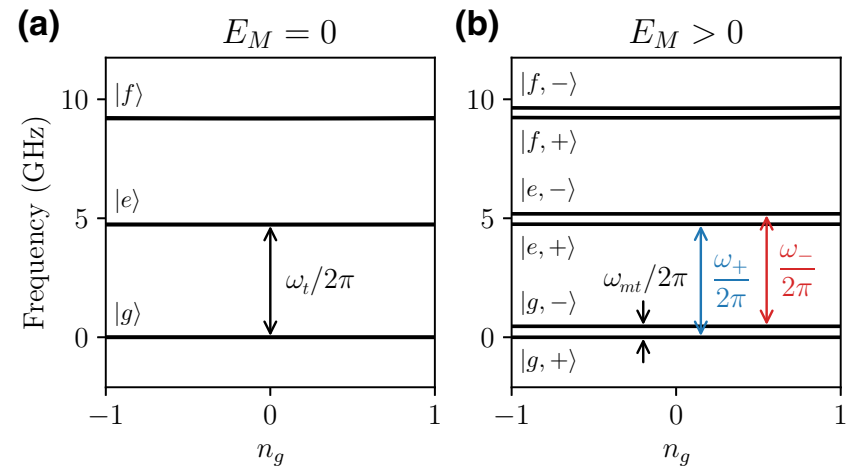

FIG. 2. The low-level spectrum of a Majorana transmon qubit as a function of the offset charge parameter $n_{g}$, where $E_{C} / h=$ $250 \mathrm{MHz}, E_{J} / E_{C}=50\left(\omega_{t} / 2 \pi \simeq 5 \mathrm{GHz}\right)$, and (a) $E_{M}=0$ and (b) $E_{M} / \hbar \omega_{t}=0.05\left(\omega_{m t} / \omega_{t} \simeq 0.1\right)$. When $E_{M}=0$, each level in the transmon spectrum is two-fold degenerate. This degeneracy is lifted when $E_{M}>0$. The resulting level structure can be thought of as two parallel transmon ladders, for $i \hat{\gamma}_{2} \hat{\gamma}_{3}= \pm 1$.

where $\left[\hat{b}, \hat{b}^{\dagger}\right]=1$. Taylor expanding the $\cos \hat{\varphi}$ term to fourth order in $\hat{\varphi}$, substituting the expressions above, and dropping fast rotating terms, we obtain the standard result

$$
\hat{H}_{T} \simeq \hbar \omega_{t} \hat{b}^{\dagger} \hat{b}-\frac{E_{C}}{2} \hat{b}^{\dagger} \hat{b}^{\dagger} \hat{b} \hat{b}
$$

where $\hbar \omega_{t}=\sqrt{8 E_{J} E_{C}}-E_{C}$ is the transmon energy and $E_{C}$ is the anharmonicity.

Repeating the steps for the Majorana term $\hat{H}_{M}$ yields

$$
\hat{H}_{M} \simeq-i E_{M} \hat{\gamma}_{2} \hat{\gamma}_{3}\left(1-\xi_{0}-\xi_{1} \hat{b}^{\dagger} \hat{b}+\xi_{2} \hat{b}^{\dagger} \hat{b}^{\dagger} \hat{b} \hat{b}\right),
$$

with coefficients

$$
\begin{aligned}
& \xi_{0}=\frac{4 \sqrt{8 E_{J} E_{C}}-E_{C}}{64 E_{J}}, \\
& \xi_{1}=\frac{4 \sqrt{8 E_{J} E_{C}}-2 E_{C}}{32 E_{J}}, \\
& \xi_{2}=\frac{E_{C}}{32 E_{J}} .
\end{aligned}
$$

Under the above approximations, we see that the energy splitting of the two lowest energy levels with unequal Majorana parity, $|g, \pm\rangle$, which we label $\hbar \omega_{m t}$, is

$$
\hbar \omega_{m t} \simeq 2 E_{M}\left(1-\xi_{0}\right) .
$$

On the other hand, the "transmon transitions" $|g,+\rangle \leftrightarrow$ $|e,+\rangle$ and $|g,-\rangle \leftrightarrow|e,-\rangle$ (indicated in Fig. 2) have energy splittings

$$
\hbar \omega_{ \pm} \simeq \hbar \omega_{t} \pm E_{M} \xi_{1},
$$

respectively. As we will show, despite that there is no charge matrix element between the two logical qubit states 
$|g, \pm\rangle$, the fact that the two transition frequencies $\omega_{ \pm}$ are nondegenerate for $E_{M}>0$ nevertheless leads to a $i \hat{\gamma}_{2} \hat{\gamma}_{3}$-dependent dispersive shift of the resonator.

\section{B. Dispersive interaction with a resonator}

The Majorana transmon qubit can be read out via a resonator that is capacitively coupled to the island charge, as schematically illustrated in Fig. 1. This interaction has the form

$$
\hat{H}_{\text {int }}=i \hbar \lambda \hat{N}\left(\hat{a}^{\dagger}-\hat{a}\right)
$$

while the resonator Hamiltonian is given by $\hat{H}_{r}=\hbar \omega_{r} \hat{a}^{\dagger} \hat{a}$. Here $\hbar \lambda \simeq 2\left(C_{c} / C_{r}\right) E_{C} \sqrt{R_{K} / 4 \pi Z_{r}}$ quantifies the capacitive coupling strength, with $C_{c}$ the coupling capacitance, $C_{r}$ the resonator capacitance, $Z_{r}=\sqrt{L_{r} / C_{r}}$ the resonator characteristic impedance and $R_{K}=h / e^{2}$ the resistance quantum.

We numerically diagonalize the full Hamiltonian $\hat{H}_{\mathrm{MT}}$, Eq. (6), to calculate the dispersive shift $\chi_{m t}$ defined in Eq. (A5). To assist with interpreting our results, we first calculate dispersive shifts for a conventional transmon qubit, which corresponds to the limit $E_{M}=0$.

Conventional transmon.- In this case, the qubit is encoded in the two eigenstates $|0\rangle \equiv|g, a\rangle$ and $|1\rangle \equiv|e, a\rangle$ where the choice of $a= \pm$ is arbitrary. The spectrum is shown in Fig. 2(a). There are two primary transitions that contribute to the conventional transmon dispersive shift $\chi_{t}$, defined in Eq. (A5). Namely, the qubit transition $|g, a\rangle \leftrightarrow$ $|e, a\rangle$ with frequency $\omega_{t}$ and the transition $|e, a\rangle \leftrightarrow|f, a\rangle$ with frequency approximately given by $\omega_{t}-E_{C} / \hbar$. We numerically calculate $\chi_{t}$ as a function of the detuning parameter $\Delta_{t} \equiv \omega_{t}-\omega_{r}$ in Fig. 3(b). The singularities at $\Delta_{t}=0$ and $\Delta_{t}=E_{C} / \hbar$ correspond to values of $\omega_{r}$ where the resonator is resonant with the $|g, a\rangle \leftrightarrow|e, a\rangle$ and $|e, a\rangle \leftrightarrow|f, a\rangle$ transitions, respectively. The regime between these two singularities, where the dispersive shift changes sign, is known as the straddling regime [51]. The transmon dispersive shift $\chi_{t}$ provides a point of comparison that we use to evaluate the dispersive shifts of Majorana transmons.

Majorana transmon.-We next consider the Majorana transmon qubit with $E_{M}>0$. For the Majorana transmon qubit, the logical qubit states are chosen to be $|0\rangle \equiv$ $|g,+\rangle$ and $|1\rangle \equiv|g,-\rangle$ instead. The qubit-resonator interaction does not induce transitions between levels with different parity $a$. Instead, the contributions to the qubitstate-dependent dispersive shift $\chi_{m t}$, in analogy to $\chi_{t}$, come from transitions $|g,+\rangle \leftrightarrow|e,+\rangle$ with frequency $\omega_{+}$and $|g,-\rangle \leftrightarrow|e,-\rangle$ with frequency $\omega_{-}$, as indicated in Fig. 2 . The frequencies $\omega_{ \pm}$are close to $\omega_{t}$, approximately given by Eq. (12). As $2 E_{M} \simeq \hbar \omega_{m t}$ increases and becomes comparable to $\hbar \omega_{t}, \chi_{m t}$ approaches a comparable magnitude to $\chi_{t}$, the dispersive shift for a conventional transmon, as
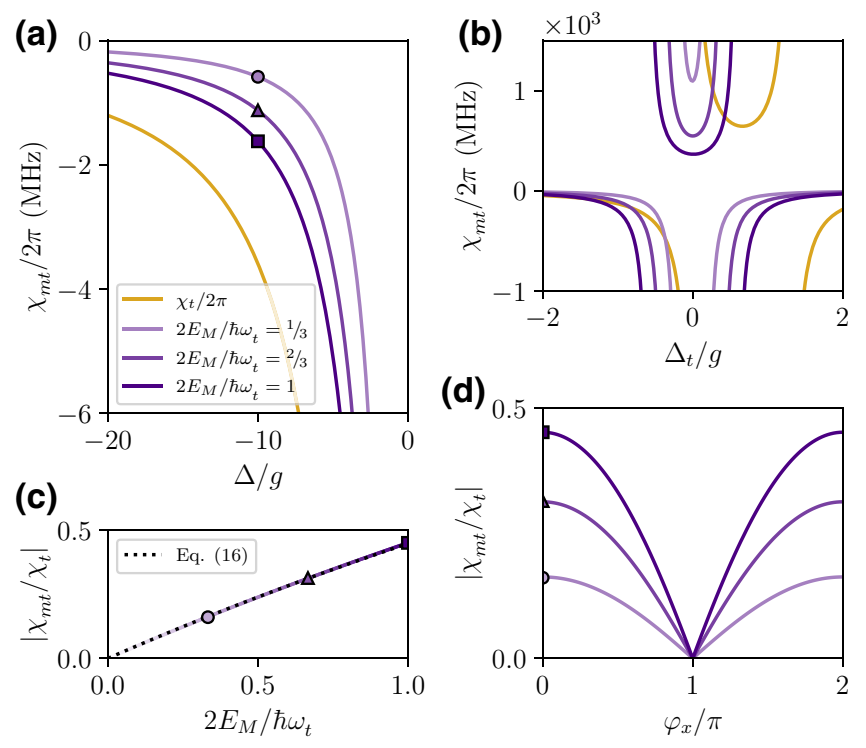

FIG. 3. Dispersive frequency shift of a readout resonator coupled to a Majorana transmon qubit $\chi_{m t}$ (purple) in comparison to those of a conventional transmon qubit $\chi_{t}$ (gold), where $E_{C} / h=250 \mathrm{MHz}, E_{J} / E_{C}=50\left(\omega_{t} / 2 \pi \simeq 5 \mathrm{GHz}\right), n_{g}=0$, and $\lambda / 2 \pi=100 \mathrm{MHz}\left(g_{t} / 2 \pi \simeq 200 \mathrm{MHz}\right)$. In (a),(b),(c) $\varphi_{x}=0$ and in (b),(c) $\Delta / g_{t}=-10$. Circle, triangle, and square markers indicate equivalent points between plots. The top plots show $\chi_{m t}$ and $\chi_{t}$ as a function of (a) $\Delta / g_{t}$ and (b) $\Delta_{t} / g_{t}$ for three values of $E_{M}$ where $\varphi_{x}=0$. In (a) $\Delta$ is the detuning from the relevant transition frequency $\left(\omega_{t}\right.$ for the transmon and $\omega_{+}$for the Majorana transmon). In (b) $\Delta_{t}=\omega_{t}-\omega_{r}$, plotted such that the saddle regimes are visible. The magnitude of $\chi_{m t}$ in comparison to $\chi_{t}$ is plotted as a function of (c) $2 E_{M} / \hbar \omega_{t}\left(\simeq \omega_{m t} / \omega_{t}\right)$ and (d) $\varphi_{x} / \pi$.

shown in Figs. 3(a) and 3(c). We note that the strength of the dispersive shift $\chi_{m t}$ also depends on the offset flux $\varphi_{x}$, as shown in Fig. 3(d). Care must be taken to ensure that $\varphi_{x} \neq \pi$, where $\chi_{m t}$ vanishes and changes sign.

We can also find an approximate analytical expression for the dispersive shift. To this end, we substitute Eq. (7) into $\hat{H}_{\text {int }}$ and make a rotating-wave approximation to find that

$$
\hat{H}_{\mathrm{int}} \simeq \hbar g_{t}\left(\hat{b} \hat{a}^{\dagger}+\hat{a} \hat{b}^{\dagger}\right)
$$

where

$$
g_{t}=\lambda\left(\frac{E_{J}}{2 E_{C}}\right)^{1 / 4}
$$

This form clearly shows how energy exchange with the resonator leads to transitions between transmon levels within the same parity sector $(|g, \pm\rangle \leftrightarrow|e, \pm\rangle,|e, \pm\rangle \leftrightarrow$ $|f, \pm\rangle$, etc.). From Eq. (14) we find the following simple approximate expression for $\chi_{m t}$ (see Appendix C):

$$
\chi_{m t} \simeq \frac{1}{2}\left(\frac{g_{t}^{2}}{\omega_{+}-\omega_{r}}-\frac{g_{t}^{2}}{\omega_{-}-\omega_{r}}\right) .
$$


This is compared to the result based on exact diagonalization of the qubit Hamiltonian in Fig. 3(c). We emphasize that Eq. (16) is a somewhat crude approximation similar in accuracy to the standard approximation used for conventional transmon qubits [see Eq. (3.12) of Ref. [51] ].

\section{MAJORANA BOX QUBIT}

\section{A. Model for the qubit}

The Majorana box qubit, shown in Fig. 1(b), is an alternative design for a qubit based on MZMs that has been studied in the context of measurement-only topological quantum computing $[25,26]$. In this qubit design, the topological superconductors are shunted by a (trivial) superconducting bridge instead of a Josephson junction. This model can be thought of as a limiting case to the Majorana transmon, where $E_{J} / E_{C} \rightarrow \infty$. In this limit we have $\hat{\varphi}_{R} \rightarrow \hat{\varphi}_{L}$, such that the previous charge and phase operators, $\hat{N}=\left(\hat{N}_{L}-\hat{N}_{R}\right) / 2$ and $\hat{\varphi}=\hat{\varphi}_{L}-\hat{\varphi}_{R}$, are zero. Instead, the relevant degree of freedom is the total charge $\hat{N}_{\text {tot }}=\hat{N}_{L}+\hat{N}_{R}$, and the corresponding conjugate phase $\hat{\varphi}_{\text {tot }} \equiv\left(\hat{\varphi}_{L}+\hat{\varphi}_{R}\right) / 2$. The device acts as a single island with charging energy

$$
\hat{H}_{\text {tot }}=E_{\text {tot }}\left(\hat{N}_{\text {tot }}-n_{g}\right)^{2},
$$

where $E_{\text {tot }}$ quantifies the charging energy due to capacitive coupling of the island to ground (and to the resonator), which we neglected for the Majorana transmon. The charge and phase operators $\hat{N}_{\text {tot }}, \hat{\varphi}_{\text {tot }}$ act on charge eigenstates analogously to Eq. (4), where $\hat{N}_{\text {tot }}$ now counts the total charge on the superconducting island consisting of the two topological superconductors.

The Majorana box qubit also comes in several qualitatively similar variations. When the two topological superconductors are aligned horizontally in series as in Fig. 1(b) (formed from a single nanowire), the qubit is also refereed to as a Majorana loop qubit. Alternatively, the two topological superconductors can be arranged in parallel with the superconducting shunt perpendicular to the nanowires [25]. One can also consider additional MZMs per island, used as ancilla modes for measurement-only topological quantum computing. In this case, a Majorana box qubit with four MZMs is called a tetron, with six MZMs a hexon, and so on. Our results can be generalized to these variations.

The coupling of the two topological superconductors due to a nonzero overlap of the Majorana modes corresponding to $\hat{\gamma}_{2}$ and $\hat{\gamma}_{3}$ can be modeled using Eq. (5) with $\hat{\varphi} \rightarrow 0$. However, to properly account for the movement of charge that leads to a coupling to the resonator, we take one step back and explicitly include coupling to bound states in the semiconducting region between the two topological superconductors. In the limit of where the energy penalty to occupy these bound states is large compared to the tunnel coupling, the Hamiltonian $\hat{H}_{M}$ can be recovered as an effective description. Including such bound states as intermediate degrees of freedom is however necessary to correctly capture the coupling to the resonator that results from charge tunneling to the semiconductor. In the proposals in Refs. [25,26], this description is moreover very natural because a gate-defined quantum dot is explicitly introduced to mediate a tunable interaction between the nanowires.

We model the quantum dot between the two topological superconductors by a single fermionic operator $\hat{d}$, satisfying $\left\{\hat{d}, \hat{d}^{\dagger}\right\}=1$. This degree of freedom is illustrated in Fig. 1(b). The dot is described by a Hamiltonian $\hat{H}_{d}=$ $\varepsilon \hat{d}^{\dagger} \hat{d}$ with $\varepsilon$ the dot occupation energy. Tunneling between the island and the dot is modeled by a Hamiltonian [56]

$$
\hat{H}_{t}=\frac{1}{2}\left[e^{i \hat{\varphi}_{\mathrm{tot}} / 2}\left(i t_{L} e^{i \varphi_{x} / 2} \hat{\gamma}_{2}-t_{R} \hat{\gamma}_{3}\right) \hat{d}+\text { H.c. }\right] \text {, }
$$

where $t_{L, R} \geq 0$ are the tunneling amplitudes between the two respective topological superconductors, and we have included the possibility of an external flux, $\varphi_{x}$, threading the qubit loop. The full Majorana box qubit Hamiltonian is thus

$$
\hat{H}_{\mathrm{MB}}=\hat{H}_{\mathrm{tot}}+\hat{H}_{d}+\hat{H}_{t} .
$$

We show the spectrum in the uncoupled case $t_{L, R}=0$ in Fig. 4(a). As with the Majorana transmon qubit, each state shown in Fig. 4(a) is two-fold degenerate, a degeneracy that splits when we include nonzero tunneling $\left|t_{L, R}\right|>0$, as shown in Fig. 4(b).

The dot-island Hamiltonian $\hat{H}_{\mathrm{MB}}$ conserves the total charge $\hat{N}_{\text {tot }}+\hat{d}^{\dagger} \hat{d}$, such that the Hamiltonian can be diagonalized block by block, following Ref. [30]. After a unitary transformation $\hat{H}_{\mathrm{MB}}^{\prime}=\hat{U}^{\dagger} \hat{H}_{\mathrm{MB}} \hat{U}$ we have

$$
\begin{aligned}
\hat{H}_{\mathrm{MB}}^{\prime}= & \varepsilon_{c}(\hat{N}+1) \hat{d}^{\dagger} \hat{d}+\frac{\varepsilon_{m}(\hat{N})}{2} i \hat{\gamma}_{2} \hat{\gamma}_{3} \\
& +\frac{1}{2}\left[\varepsilon_{m}(\hat{N}+1)-\varepsilon_{m}(\hat{N})\right] \hat{d}^{\dagger} \hat{d} \hat{i} \hat{\gamma_{2}} \hat{\gamma}_{3} \\
& +E(\hat{N})+[E(\hat{N}+1)-E(\hat{N})] \hat{d}^{\dagger} \hat{d} .
\end{aligned}
$$

The functions $\varepsilon_{c}(n), \varepsilon_{m}(n)$, and $E(n)$ are given in Eq. (D9).

As is clear from Eq. (20), the eigenstates of $\hat{H}_{\mathrm{MB}}^{\prime}$ can be labeled by three quantum numbers $\left|N, n_{d}, a\right\rangle$ : the island charge $N \in \mathbb{Z}$, the dot occupancy $n_{d}=0,1$, and the Majorana parity $a= \pm$. The dressed eigenstates of $\hat{H}_{\mathrm{MB}}$, Eq. (19), are thus related to the bare charge states of the uncoupled system (i.e., when $t_{L, R}=0$ ) through

$$
\overline{\left|N, n_{d}, a\right\rangle}=\hat{U}\left|N, n_{d}, a\right\rangle
$$



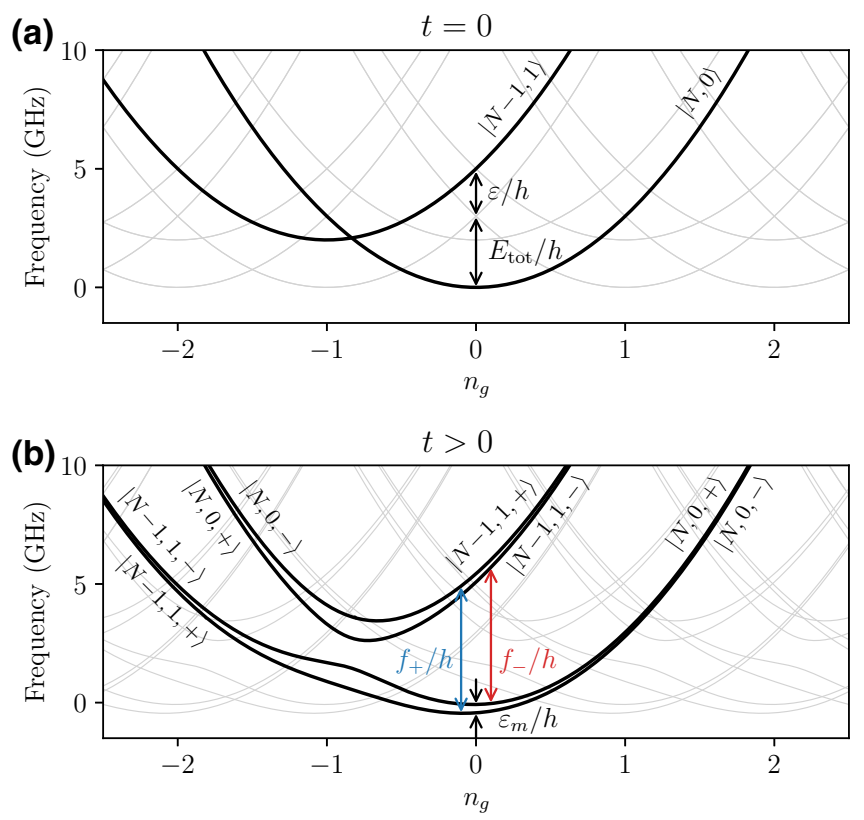

FIG. 4. The low-level spectrum of a Majorana box qubit as a function of the offset charge parameter $n_{g}$, where $\delta / h=\left(E_{\mathrm{tot}}+\right.$ $\varepsilon) / h=5 \mathrm{GHz}, \varphi_{x}=\pi / 2, t_{L, R}=t$, and (a) $t=0$ and (b) $t / \delta \simeq$ $0.2\left(\varepsilon_{m} / h \simeq 0.5 \mathrm{GHz}\right)$. When MZMs interact via the quantum dot $(t>0)$, the charge state $\left|N, n_{d}=0, \pm\right\rangle$ hybridizes with $\mid N-$ $\left.1, n_{d}=1, \mp\right\rangle$.

where the unitary transformation is defined in Appendix D 1. The labels on the left-hand side here designate hybridized degrees of freedom, in particular, when $t_{L, R}>0$, the Majorana fermion hybridizes with the dot, and $a= \pm$ refers to the corresponding "dressed" Majorana parity.

To keep the discussion simple, from now on we focus on the sector with zero total dot-island charge, $\hat{N}+\hat{d}^{\dagger} \hat{d}=0$, and set $t_{L, R}=t, n_{g}=0$ for the remainder of this section. Within the zero total charge sector, Eq. (20) takes the form

$$
\hat{H}_{\mathrm{MB}, 0}=\varepsilon_{c} \hat{c}^{\dagger} \hat{c}+\frac{\varepsilon_{m}}{2} i \hat{\gamma}_{2} \hat{\gamma}_{3}
$$

where we have dropped a constant term, and $\hat{c}$ satisfies $\left\{\hat{c}, \hat{c}^{\dagger}\right\}=1$ and describes the movement of an electron from the dot to the island within the zero total charge sector. The coefficients are given by

$$
\begin{aligned}
\varepsilon_{c} & =\frac{1}{2}\left(f_{+}+f_{-}\right), \\
\varepsilon_{m} & =\frac{1}{2}\left(f_{+}-f_{-}\right), \\
f_{ \pm} & =\sqrt{\delta^{2}+2 t^{2}\left[1 \pm \cos \left(\varphi_{x} / 2\right)\right]},
\end{aligned}
$$

where $\delta=E_{\text {tot }}+\varepsilon$ is the energy penalty for moving charge from the island to the dot. For small $t / \delta$, the second term in Eq. (22) moreover reduces to Eq. (5), since

$$
\varepsilon_{m} \simeq \frac{t^{2}}{\delta} \cos \left(\frac{\varphi_{x}}{2}\right)
$$

In the opposite limit, if the chemical potential of the quantum dot is tuned such that $\varepsilon=-E_{\text {tot }}$ and the energy penalty to occupy the $\operatorname{dot} \delta=0$ then $\varepsilon_{m} \sim t$.

\section{B. Dispersive interaction with a resonator}

As with the Majorana transmon, we can read out the logical state by coupling the qubit to a resonator. There are essentially two options for engineering a dipole coupling by capacitively coupling to the resonator. Either the resonator voltage can be (predominantly) coupled to the dot or (predominantly) to the superconducting island. The key requirement for readout is that the resonator must be sensitive to the movement of charge between the superconducting island and the dot, and the two coupling schemes are in that sense equivalent (as shown, e.g., in Ref. [30]). The two choices might, however, have different practical advantages and disadvantages; in particular, stronger coupling may be possible by coupling to the island. We here focus mainly on capacitive coupling to the superconducting island charge for concreteness, but we emphasize that our results apply equally well to both schemes. The qubitresonator interaction is thus, in analogy with Eq. (13), given by

$$
\hat{H}_{\text {int }}=i \hbar \lambda \hat{N}_{\text {tot }}\left(\hat{a}^{\dagger}-\hat{a}\right) .
$$

We perform the same unitary transformation that led to Eq. (22), and again set $t_{L, R}=t, n_{g}=0$ and project onto the subspace with zero overall charge, to find an interaction in this subspace of the form (see Appendix D 1)

$$
\begin{aligned}
\hat{H}_{\text {int }, 0}= & i \hbar\left[g_{c} \hat{c}^{\dagger} \hat{c}+\frac{g_{m}}{2}\left(i \hat{\gamma}_{2} \hat{\gamma}_{3}+1\right)\right]\left(\hat{a}^{\dagger}-\hat{a}\right) \\
& +\frac{i \hbar}{2}\left[g_{+}\left(\hat{\gamma}_{2}+i \hat{\gamma}_{3}\right) \hat{c}+\text { H.c. }\right]\left(\hat{a}^{\dagger}-\hat{a}\right) \\
& +\frac{i \hbar}{2}\left[g_{-}\left(\hat{\gamma}_{2}-i \hat{\gamma}_{3}\right) \hat{c}+\text { H.c. }\right]\left(\hat{a}^{\dagger}-\hat{a}\right),
\end{aligned}
$$

where

$$
\begin{aligned}
g_{c} & =-\frac{\lambda}{2}\left(\frac{\delta}{f_{+}}+\frac{\delta}{f_{-}}\right), \\
g_{m} & =-\frac{\lambda}{2}\left(\frac{\delta}{f_{+}}-\frac{\delta}{f_{-}}\right), \\
g_{ \pm} & =-\frac{\lambda}{2} \frac{i t\left(e^{i \varphi_{x} / 2} \pm 1\right)}{f_{ \pm}} .
\end{aligned}
$$

We note that, in the case where the quantum dot is resonant with the island and $\delta=0$, then $g_{c}=g_{m}=0$ and $\left|g_{ \pm}\right|=$ 
$\lambda / 2$. For the alternative choice of coupling the resonator to the dot, simply replace $\hat{N}_{\text {tot }} \rightarrow \hat{d}^{\dagger} \hat{d}$ in Eq. (25) and the above results still apply with a sign change $\lambda \rightarrow-\lambda$ in Eq. (27) [30].

From the second and third lines of Eq. (26) we see that, in this frame, the resonator induces a transition that involves moving an electron from the dot to the island and flipping the Majorana parity $i \hat{\gamma}_{2} \hat{\gamma}_{3}$. The energy difference corresponding to this transition is $\varepsilon_{c} \pm \varepsilon_{m}=f_{ \pm}$, depending on the state of the Majorana degree of freedom.

Having diagonalized the Majorana box qubit Hamiltonian, it is straightforward to use the second-order Schrieffer-Wolff formula Eq. (A4) to obtain an analytical expression for the qubit-state-dependent dispersive shift. Under a rotating-wave approximation for the resonatorqubit interaction, we find that

$$
\chi_{m b} \simeq \frac{1}{2}\left(\frac{\left|g_{+}\right|^{2}}{f_{+} / \hbar-\omega_{r}}-\frac{\left|g_{-}\right|^{2}}{f_{-} / \hbar-\omega_{r}}\right),
$$

where we assume that $\varepsilon_{c}>\varepsilon_{m}$.

We compare Eq. (28) to a numerical diagonalization of the qubit Hamiltonian, following the same procedure as for the Majorana transmon qubit, to extract the qubitdependent dispersive shift $\chi_{m b}$. In Fig. 5(a) we show $\chi_{m b}$ as a function of $\Delta /\left|g_{+}\right|$for different tunneling strengths $t_{L, R}=t$, where $\Delta \equiv f_{+} / \hbar-\omega_{r}$. As the Majorana modes (a)

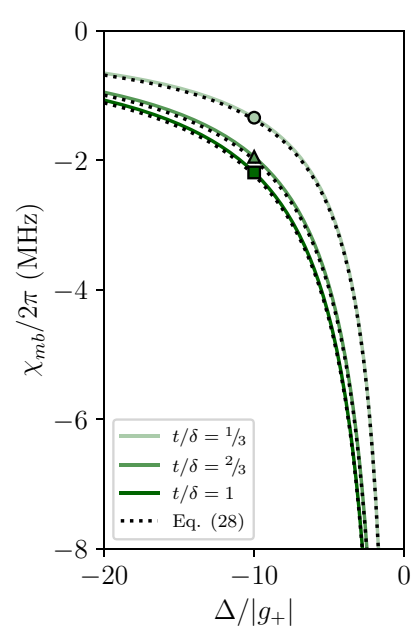

(b)
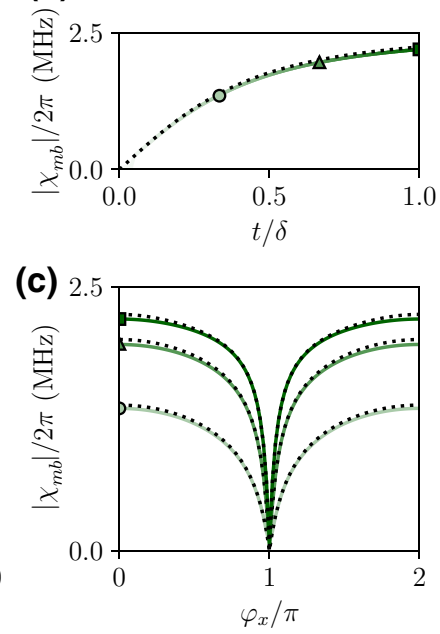

FIG. 5. Dispersive frequency shifts of a readout resonator coupled to a Majorana box qubit $\chi_{m b}$ (green) for $\delta / h=\left(E_{\text {tot }}+\right.$ $\varepsilon) / h=5 \mathrm{GHz}, n_{g}=0, t_{L, R}=t$, and $\lambda / 2 \pi=100 \mathrm{MHz}$. In (a),(b) $\varphi_{x}=0$ and in (b),(c) $\Delta / g_{+}=-10$. Circle, triangle, and square markers indicate equivalent points between plots. We show $\chi_{m b}$ as a function of (a) detuning $\Delta=f_{+} / \hbar-\omega_{r}$ for three values of $t / \delta$, (b) $t / \delta$ for $\Delta /\left|g_{+}\right|=-10$, (c) $\varphi_{x}=0$. Analytical results (dotted) obtained from Eq. (28) are also shown. A very small discrepancy arises due to the rotating-wave approximation used in Eq. (28). hybridize with the dot, the qubit splitting and the dispersive shifts grow larger, also shown in Fig. 4(b). As with the Majorana transmon, the dispersive shift $\chi_{m b}$ depends on the offset flux $\varphi_{x}$, as shown in Fig. 5(c). Again, care must be taken to ensure that $\varphi_{x} \neq \pi$. However, in contrast to the Majorana transmon, the dependence is more favorable, leading to a wider range of $\varphi_{x}$ where $\chi_{m b}$ is close to its maximum possible magnitude.

Our results show that the energy scale of the dispersive shifts for the Majorana box qubit are comparable to the Majorana transmon qubit for comparable ratios between resonator coupling strengths and detuning $\Delta / g$. A more detailed comparison is made in Sec. V.

\section{READOUT TIMES AND FIDELITIES}

In this section we calculate estimates of the timescales and fidelities of the Majorana qubit readout schemes presented in the previous sections. The key results are presented in Fig. 6. It is important to note that these estimates have been obtained for an idealized situation where the dispersive approximation is assumed to be valid, and no noise or decoherence is included beyond the dephasing caused by the measurement itself. These results are therefore not meant to be quantitative predictions for the measurement fidelity in an experiment, but serve to compare the speed and fidelity for different qubit types: the conventional transmon, Majorana transmon, and the Majorana box qubit. For the Majorana box qubit, we also compare dispersive readout to longitudinal readout; see Appendix E.

Our predictions of the dispersive shifts of the Majorana transmon and Majorana box qubit as functions of qubit frequency $\omega_{q}$ are compared in Fig. 6(a). To make this comparison, we fix the value of $\Delta / g=-10$, where $g \in\left\{g_{t}, g_{+}\right\}$[see Eqs. (15) and (27c)] and $\Delta \in\left\{\omega_{+}-\right.$ $\left.\omega_{r}, f_{+} / \hbar-\omega_{r}\right\}$ [see Eqs. (12) and (23c)] for the Majorana transmon and the Majorana box qubit, respectively. We also include the dispersive shift of a conventional transmon, for which we use $g=g_{t}$ and $\Delta=\omega_{t}-\omega_{r}$. In other words, $g$ and $\Delta$ quantify the relevant coupling strength and resonator detuning for each qubit type, respectively.

We observe that, for smaller qubit frequencies (corresponding to weaker MZM interaction energies), the Majorana box qubit produces larger dispersive shifts than the Majorana transmon for the same value of $\Delta / g$. Nevertheless, both variants may achieve dispersive shifts in the megahertz regime for reasonable parameters, comparable to conventional transmon qubits [41] and the recent demonstration of a nanowire quantum dot readout in Ref. [44].

The qubit-state-dependent phase shift that arises during dispersive coupling allows for readout of the qubit by probing the resonator at its resonant frequency. The size of the dispersive shift $\chi$ directly determines the rate at which this 
(a)

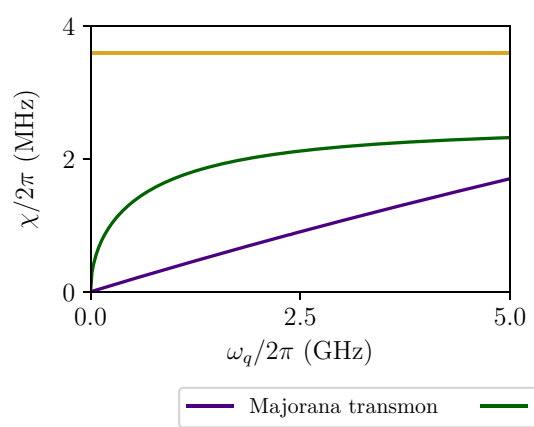

(b)

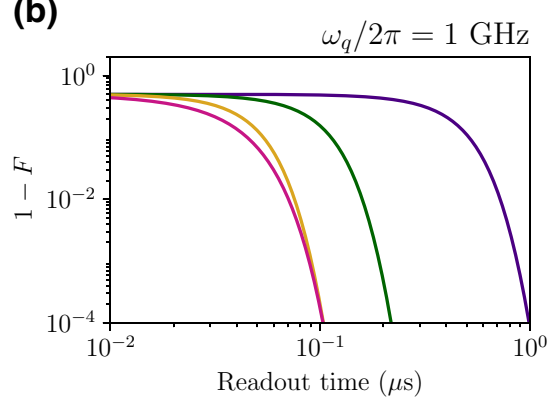

(c)

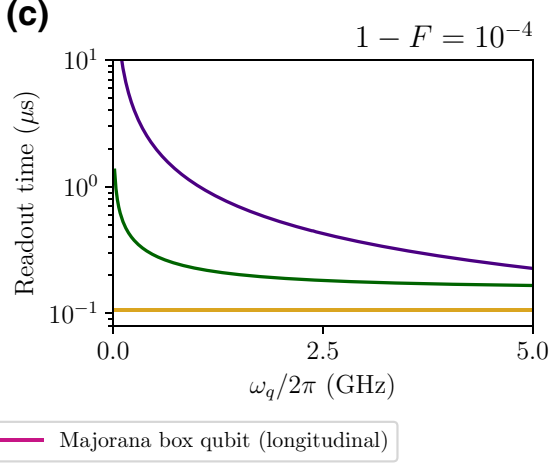

FIG. 6. Timescales and fidelities for resonator-based readout of Majorana qubits. (a) Dispersive shift of a readout resonator coupled to a Majorana transmon qubit $\chi_{m t}$ (purple), a Majorana box qubit $\chi_{m b}$ (green), and a conventional transmon $\chi_{t}$ (gold) as a function of the qubit frequency $\omega_{q} / 2 \pi$ for $n_{g}=0, \varphi_{x}=0, \lambda / 2 \pi=100 \mathrm{MHz}$, and fixed detuning $\Delta / g=-10$. For the Majorana and conventional transmon we have set $E_{C} / h=250 \mathrm{MHz}$ and $E_{J} / E_{C}=50$. For the Majorana box qubit, we have set $\delta / h=\left(E_{\text {tot }}+\varepsilon\right) / h=5 \mathrm{GHz}$. (b) Infidelity $1-F$ of each readout scheme as a function of readout time $\tau$ for $\omega_{q} / 2 \pi=1 \mathrm{GHz}, \bar{n} / n_{\text {crit }}=1 / 5$, and $\kappa=2 \chi$. Longitudinal case calculated for $\tilde{g}_{z} / 2 \pi=10 \mathrm{MHz}$, as detailed in Appendix E. (c) Readout time required to reach a measurement fidelity of $99.99 \%$ for each dispersive scheme in (a) as a function of $\omega_{q} / 2 \pi$.

phase shift can be resolved to a given fidelity. We quantify this effect with the signal-to-noise ratio (SNR) for a heterodyne measurement of the resonator output field. To simplify the treatment, we consider an idealized situation with unit efficiency measurement and no additional noise or decoherence, such that the qubit-dependent response of the resonator is Gaussian. In this case an analytical form for the SNR can be found [39,57]:

$$
\mathrm{SNR}=2|\epsilon| \sqrt{\frac{\tau}{\chi}}\left[1-\frac{1}{\chi \tau}\left(1-e^{-\chi \tau} \cos \chi \tau\right)\right] .
$$

Here $|\epsilon|$ is the amplitude of the resonator drive and $\tau$ is the measurement time. The SNR will in general depend on the resonator damping rate $\kappa$, and we have set $\kappa=2 \chi$ to give the optimal SNR at long integration times [39,57].

The assignment fidelity can be related to the SNR through $[30,39]$

$$
F=1-\frac{1}{2}[p(1 \mid 0)+p(0 \mid 1)]=1-\frac{1}{2} \operatorname{erfc}\left(\frac{\mathrm{SNR}}{2}\right),
$$

where $p(i \mid j)$ is the probability of obtaining the outcome $i$ given that the qubit was in the state $|j\rangle$. (We note that an alternative definition of fidelity $F_{m}=1-p(1 \mid 0)-$ $p(0 \mid 1)=2 F-1$ is also often used [39].) We emphasize that these results hold for the ideal, dispersive Hamiltonian Eq. (2). In other words, the dispersive approximation is assumed to be valid. This approximation will break down for large photon numbers [39].

From these expressions, we calculate the expected measurement infidelities $1-F$ for each qubit as a function of integration time $\tau$ at $\omega_{q} / 2 \pi=1 \mathrm{GHz}$ in Fig. 6(b). We have chosen the resonator drive strength $\epsilon$ such that $\bar{n} / n_{\text {crit }}=1 / 5$, where $\bar{n}=2(\epsilon / \kappa)^{2}$ is the resonator photon number and $n_{\text {crit }} \equiv(\Delta / 2 g)^{2}$. The latter can be thought of as a rough measure of when the dispersive approximation is expected to break down [39].

For comparison, we also show the infidelity of a longitudinal readout scheme for the Majorana box qubit in Fig. 6(b). We have chosen parameters such that $\kappa$ and $\bar{n}$ are equal between the dispersive and longitudinal cases, which for these parameters correspond to a modulation of the longitudinal coupling strength by $\tilde{g}_{z} / 2 \pi \simeq 10 \mathrm{MHz}$. As shown in Fig. 8 in Appendix E such a modulation can be achieved by a very modest modulation in either the tunnel coupling or external flux. It is noteworthy that longitudinal readout gives a much faster (and thus higher fidelity) readout for this modest value of parametric modulation. For example, doubling the modulation amplitude translates to a readout that is roughly twice as fast.

Finally, we calculate the measurement integration time required to achieve a measurement fidelity of $99.99 \%$ for the dispersive readout protocols as a function of qubit splitting $\omega_{q}$, shown in Fig. 6(d). For the chosen system parameters and assumptions, both Majorana qubits may achieve high-fidelity dispersive measurements in a fraction of a microsecond. Furthermore, the Majorana box qubit, which produces a larger dispersive shift, benefits from a faster readout time at the same value of $\Delta / g$ and qubit frequency.

\section{CONCLUSIONS}

Our results are very promising for dispersive readout as a means to measure Majorana qubits quickly and with high fidelity. We have calculated the qubit-dependent dispersive shifts of a readout resonator for Majorana transmons and Majorana box qubits, under a simple capacitive coupling 
of the resonator to the qubit. This dispersive shift can be used to readout the state of the qubit by measuring the phase shift of a resonant probe tone on the resonator. We find that the dispersive shift for Majorana qubits of both types can be in the megahertz range for reasonable parameters. These results are encouraging, as they indicate that well-established and extremely successful readout techniques can be adopted from the circuit QED context $[39,41]$.

There are some key differences in the QND nature of dispersive readout for a Majorana transmon compared to a Majorana box qubit. For the Majorana transmon, the qubitresonator interaction manifestly preserves the Majorana parity, independent of the detuning of the readout resonator from the relevant transitions between qubit energy levels. This protection originates from the fact that both $\hat{H}_{\mathrm{MT}}$ in Eq. (6) and $\hat{H}_{\text {int }}$ in Eq. (13) commute with $i \hat{\gamma}_{2} \hat{\gamma}_{3}$. The Majorana parity is therefore preserved independently of whether the perturbative dispersive approximation $H_{\text {disp }}$, Eq. (2), is valid. As a result, the dispersive readout of a Majorana transmon is quantum nondemolition in a stronger sense than for conventional charge qubits.

For the Majorana box qubit, the situation is different. Here, coupling to the resonator is induced by tunneling of charge from the qubit island to a nearby quantum dot. In a readout scheme, the tunnel coupling should be turned on adiabatically, such that the system evolves into dressed joint eigenstates of the qubit-dot system [see Eq. (20)]. However, the interaction with the resonator induces transitions between dressed eigenstates of different Majorana parity; see Eq. (26). A quantum nondemolition readout is therefore only approximately recovered in a limit where the relevant transition frequency for moving an electron between the island and the dot is far detuned from the resonator frequency, leading to $\hat{H}_{\text {disp }}$ in Eq. (2). The readout is therefore no longer QND when the dispersive approximation breaks down, which can happen, e.g., for large photon numbers. It is worth noting, however, that the joint Majorana-dot parity is manifestly conserved by Eq. (26). If one can also determine the state of the dot, prior to and after the measurement, with high fidelity, it may be possible to confirm the Majorana parity, as has been discussed in Refs. [32,33,35].

The fundamental differences between the Majorana transmon and the Majorana box qubits makes a quantitative comparison of readout fidelity and speed more challenging. In Sec. V we compared the two qubits for equal qubit energy splitting, and at a fixed value of coupling strength relative to resonator detuning, $g / \Delta$, and fixed value of resonator photons relative to $n_{\text {crit }} \equiv(\Delta / 2 g)^{2}$. With this choice, our results suggest that the Majorana box qubit produces larger dispersive shifts, and may therefore enjoy a faster readout. However, because the breakdown of the dispersive interaction will manifest itself differently for the two qubits, this comparison might not be fair. In particular, the performance of dispersive readout for large photon numbers requires further study.

The two qubits will also likely have differing robustness to noise. To achieve high-fidelity readout, it is crucial that the readout time is much smaller than the coherence times of the qubit. The Majorana box qubit is expected to be more robust against quasiparticle poisoning due to the use of smaller superconducting islands [53]. Both qubits will suffer dephasing due to finite nanowire lengths [52]. Charge noise might reduce the readout SNR due to fluctuations in system parameters, thus leading to a longer readout time (see, e.g., Refs [34,35] for a more detailed discussion).

We also draw attention to the functional dependence of the dispersive shift on flux that threads the relevant loops for each respective qubit, shown in Figs. 3(d) and 5(c). With the likelihood that offset fluxes are present in the system, and given some distribution of these between different qubits, a challenge for the scalability of the (measurementbased) approaches is the requirement to locally tune the flux for each qubit in order to maximize readout fidelity. From this perspective we find that the Majorana box qubits are favourable, since flux tuning is likely only necessary for a limited number of qubits that have offsets very close to $\varphi_{x}=\pi$.

Finally, we note that the longitudinal readout protocol introduced in Ref. [30] is entirely independent of the resonator detuning, and can therefore be used in a regime where the parity breaking terms for the Majorana box qubit are negligible (corresponding to a regime where the dispersive shift is negligible). Our results moreover show that longitudinal readout may lead to even faster and higher fidelity readout in practice, given that a reasonable parametric modulation is possible.

\section{APPENDIX A: LIGHT-MATTER INTERACTION IN THE DISPERSIVE REGIME}

We here review the general theory of dispersive coupling between an arbitrary multilevel system, which we here simply refer to as an "artificial atom," and a resonator. We treat the resonator as a single mode for simplicity. In this section we provide a high-level summary of Ref. [58], and we refer the reader to that work for further details. We use this theory to calculate dispersive shifts for the two variants of Majorana qubits in Secs. III and IV.

Consider a generic artificial atom with eigenstates $|l\rangle$ and eigenenergies $\hbar \omega_{l}$, described by a Hamiltonian $\hat{H}_{a}=$ $\sum_{l} \hbar \omega_{l}|l\rangle\langle l|$. We assume that the artificial atom is coupled to a resonator described in a single-mode approximation by a Hamiltonian $\hat{H}_{r}=\hbar \omega_{r} \hat{a}^{\dagger} \hat{a}$. The atom-resonator coupling has the form $\hat{H}_{\text {int }}=i \hbar \lambda \hat{N}\left(\hat{a}^{\dagger}-\hat{a}\right)$ where $\hat{N}$ is the number operator for the artificial atom charge degree of freedom, which couples to the voltage of the resonator $\hat{V}_{r} \sim i\left(\hat{a}^{\dagger}-\hat{a}\right)$ with interaction strength $\lambda$. Expressing the 
interaction in the eigenbasis of the artificial atom, the two coupled systems are described by a Hamiltonian

$$
\begin{aligned}
\hat{H} & =\hbar \omega_{r} \hat{a}^{\dagger} \hat{a}+\sum_{l} \hbar \omega_{l}|l\rangle\langle l| \\
& +\sum_{l, l^{\prime}} \hbar g_{l, l^{\prime}}|l\rangle\left\langle l^{\prime}\right|\left(\hat{a}^{\dagger}-\hat{a}\right) .
\end{aligned}
$$

The coefficients $g_{l, l^{\prime}}=i \lambda\left\langle l|\hat{N}| l^{\prime}\right\rangle$ are matrix elements for transitions between states in the multilevel artificial atom. The dispersive regime refers to a situation where the frequency detunings between all relevant atomic transitions and the resonator, $\Delta_{l, l^{\prime}}=\omega_{l}-\omega_{l^{\prime}}-\omega_{r}$, are large relative to their respective transition amplitudes $g_{l, l^{\prime}}$. Energy exchange between the artificial atom and the resonator becomes a virtual process and we can write an effective Hamiltonian for Eq. (A1) that is derived from second-order Schrieffer-Wolff perturbation theory [58-60],

$$
\hat{H}_{\mathrm{disp}}=\hbar \omega_{r} \hat{a}^{\dagger} \hat{a}+\sum_{l} \hbar\left(\omega_{l}+\eta_{l}\right)|l\rangle\left\langle l\left|+\sum_{l} \hbar \chi_{l} \hat{a}^{\dagger} \hat{a}\right| l\right\rangle\langle l| .
$$

At this level of approximation the interaction is diagonal both with respect to the artificial atom and resonator eigenstates. Here, $\chi_{l}$ is a state-dependent frequency shift to the resonator and $\eta_{l}$ is a correction to $\omega_{l}$, given by

$$
\begin{aligned}
\chi_{l} & =\sum_{l^{\prime}} \chi_{l, l^{\prime}}-\chi_{l^{\prime}, l}, \\
\eta_{l} & =\sum_{l^{\prime}} \chi_{l, l^{\prime}}, \\
\chi_{l, l^{\prime}} & =\frac{\left|g_{l, l^{\prime}}\right|^{2}}{\Delta_{l, l^{\prime}}} .
\end{aligned}
$$

If a qubit is encoded in two states $\{|l=0\rangle,|l=1\rangle\}$ of the artificial atom then we obtain Eq. (2) with $\hat{\sigma}_{z}=|1\rangle\langle 1|-$ $|0\rangle\langle 0| \omega_{q}=\omega_{1}-\omega_{0}+\eta_{1}-\eta_{0}$, and

$$
\chi_{q}=\frac{1}{2}\left(\chi_{1}-\chi_{0}\right) .
$$

In this paper, we analyze the qubit-state-dependent dispersive frequency shift $\chi_{q}$ for $q \in\{t, m t, m b\}$, corresponding to the conventional transmon qubit, the Majorana transmon qubit, and the Majorana box qubit, respectively. The choice of logical qubit states $|l=0\rangle$ and $|l=1\rangle$ are different for each qubit: $\{|g\rangle,|e\rangle\}$ for the conventional transmon, $\{|g,+\rangle,|g,-\rangle\}$ for the Majorana transmon, and for the Majorana box qubit, the two dressed states $\left\{\overline{\left|N, n_{d},+\right\rangle}, \overline{\left|N, n_{d},-\right\rangle}\right\}$ adiabatically connected to the two degenerate ground states when the tunnel couplings are zero, $t_{L, R}=0$. We denote the corresponding dispersive shifts by

$$
\begin{aligned}
\chi_{t} & =\frac{1}{2}\left(\chi_{e}-\chi_{g}\right), \\
\chi_{m t} & =\frac{1}{2}\left(\chi_{g,-}-\chi_{g,+}\right), \\
\chi_{m b} & =\frac{1}{2}\left(\chi_{N, n_{d},+}-\chi_{N, n_{d},-}\right) .
\end{aligned}
$$

\section{APPENDIX B: MODELING INTERACTIONS BETWEEN MZMS}

In this section we demonstrate that the "direct" interaction term, Eq. (5), for a Majorana transmon is equivalent to a model where the interaction is mediated by a quantum dot, in the limit where the energy cost for moving an electron onto the dot is sufficiently large. Consider a pair of topological superconductors, such as those shown in Fig. 1(a). Adjacent MZMs $\hat{\gamma}_{2}$ and $\hat{\gamma}_{3}$ are separated by an insulating-semiconducting region. Interaction between the Majorana modes is controlled by a gate voltage, which tunes the chemical potential in the semiconducting region. When the chemical potential is sufficiently large, the region forms a potential barrier that prohibits interaction between the MZMs. However, when the height of the potential barrier is lowered, electronic bound states in the semiconducting region become energetically accessible. For simplicity, we consider a single energy level, described by fermionic operator $\hat{d}$ and Hamiltonian $\hat{H}_{d}=\varepsilon \hat{d}^{\dagger} \hat{d}$. The MZMs couple to the dot, described by a tunneling Hamiltonian [56]

$$
\hat{H}_{t}=\frac{1}{2}\left[\left(i t_{L} e^{i \hat{\varphi}_{L} / 2} \hat{\gamma}_{2}-t_{R} e^{i \hat{\varphi}_{R} / 2} \hat{\gamma}_{3}\right) \hat{d}+\text { H.c. }\right] .
$$

This "indirect" model, wherein the MZMs interact via virtual occupation of the quantum dot can be compared to a
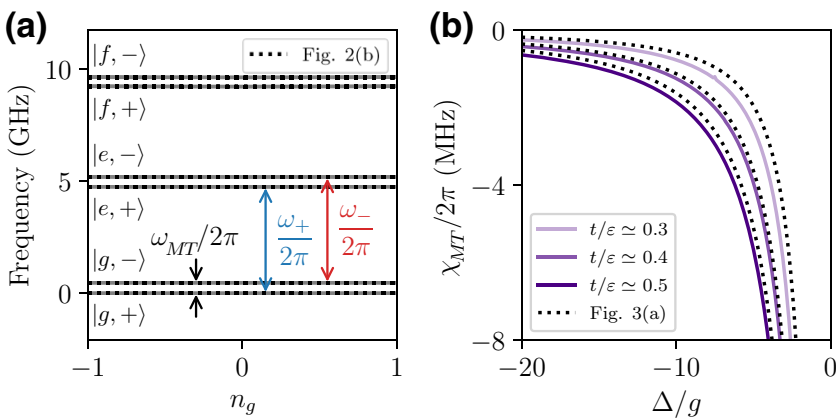

FIG. 7. Low-energy spectrum and dispersive shifts of a Majorana transmon qubit with an "indirect" interaction model. System parameters are identical to those in Fig. 3(a). Here $\varepsilon / h=20 \mathrm{GHz}$ and $t_{L, R}=t$ is tuned such that the energy splitting between the two lowest levels, $\hbar \omega_{m t}$, is equal to the corresponding case from Fig. 3(a). 
"direct" interaction, Eq. (5) [49]:

$$
\hat{H}_{M}=-i E_{M} \hat{\gamma}_{2} \hat{\gamma}_{3} \cos \left(\frac{\hat{\varphi}+\varphi_{x}}{2}\right)
$$

with $\hat{\varphi}=\hat{\varphi}_{L}-\hat{\varphi}_{R}$. The two models agree when $\delta=\varepsilon+$ $E_{C}$ is large relative to the tunnel couplings $t_{L, R}$ where $E_{C}$ is the charging energy due to capacitive coupling between the two topological superconductors, from Eq. (3). To demonstrate this, we have numerically plotted the spectrum and dispersive shifts of a Majorana transmon qubit for both interaction terms in Fig. 7.

\section{APPENDIX C: DISPERSIVE SHIFT FOR THE MAJORANA TRANSMON QUBIT}

In Sec. III B we provided an approximate formula for the dispersive frequency shift of a resonator coupled to a Majorana transmon qubit, Eq. (16). These were obtained analytically by applying the Schrieffer-Wolff method detailed in Appendix A to the complete Majorana transmon qubit-resonator Hamiltonian

$$
\hat{H}=\hat{H}_{r}+\hat{H}_{\mathrm{MT}}+\hat{H}_{\text {int }} \text {. }
$$

This treatment requires the qubit Hamiltonian

$$
\hat{H}_{\mathrm{MT}}=\hat{H}_{T}+\hat{H}_{M}
$$

to be written in a diagonal form. We use an approximation, where $\hat{H}_{T}$ and $\hat{H}_{M}$ are given in Eqs. (8) and (9), respectively. The qubit-resonator interaction Hamiltonian has the specified form

$$
\hat{H}_{\mathrm{int}}=i \hbar \lambda \hat{N}\left(\hat{a}^{\dagger}-\hat{a}\right),
$$

where we use $\hat{N}=i\left(E_{J} / 2 E_{C}\right)^{1 / 4}\left(\hat{b}^{\dagger}-\hat{b}\right)$. We proceed by calculating the dispersive frequency shift of the resonator

$$
\chi_{m t}=\frac{1}{2}\left(\chi_{g,-}-\chi_{g,+}\right)
$$

for the logical qubit states $|0\rangle=|g,+\rangle$ and $|1\rangle=|g,-\rangle$. The only nonzero matrix elements for this effective model are

$$
\begin{aligned}
& \left|g_{j, j+1}\right|=|i \lambda\langle j, a|\hat{N}| j+1, a\rangle|=g_{t} \sqrt{j+1}, \\
& \left|g_{j, j-1}\right|=|i \lambda\langle j, a|\hat{N}| j-1, a\rangle|=g_{t} \sqrt{j},
\end{aligned}
$$

where $g_{t}=\lambda\left(E_{J} / 2 E_{C}\right)^{1 / 4}$. Specifically, in the logical subspace, we have $|g,+\rangle \leftrightarrow|e,+\rangle$ with frequency $\omega_{+}$and $|g,-\rangle \leftrightarrow|e,-\rangle$ with frequency $\omega_{-}$, as indicated in Fig. 2 .
From Eq. (A3a), we obtain

$$
\begin{aligned}
\chi_{g,+} & \simeq \frac{|i \lambda\langle g,+|\hat{N}| e,+\rangle|^{2}}{-\omega_{+}-\omega_{r}}-\frac{|i \lambda\langle e,+|\hat{N}| g,+\rangle|^{2}}{\omega_{+}-\omega_{r}}, \\
& =-\frac{g_{t}^{2}}{\omega_{+}+\omega_{r}}-\frac{g_{t}^{2}}{\omega_{+}-\omega_{r}}
\end{aligned}
$$

and

$$
\begin{aligned}
\chi_{g,-} & \simeq \frac{|i \lambda\langle g,-|\hat{N}| e,-\rangle|^{2}}{-\omega_{-}-\omega_{r}}-\frac{|i \lambda\langle e,-|\hat{N}| g,-\rangle|^{2}}{\omega_{-}-\omega_{r}}, \\
& =-\frac{g_{t}^{2}}{\omega_{-}+\omega_{r}}-\frac{g_{t}^{2}}{\omega_{-}-\omega_{r}} .
\end{aligned}
$$

Finally, from Eq. (C4) we have

$$
\chi_{m t} \simeq \frac{1}{2}\left(\frac{g_{t}^{2}}{\omega_{+}-\omega_{r}}-\frac{g_{t}^{2}}{\omega_{-}-\omega_{r}}\right),
$$

where we have dropped fast-rotating terms approximately $1 /\left(\omega_{ \pm}+\omega_{r}\right)$ for simplicity.

\section{APPENDIX D: DISPERSIVE SHIFT FOR THE MAJORANA BOX QUBIT}

\section{Diagonalizing the Majorana box qubit Hamiltonian}

The complete Hamiltonian for the Majorana box qubit can be written as

$$
\begin{aligned}
\hat{H}_{\mathrm{MB}}= & E_{\mathrm{tot}}\left(\hat{N}_{\mathrm{tot}}-n_{g}\right)^{2}+\varepsilon \hat{\sigma}_{d}^{\dagger} \hat{\sigma}_{d} \\
& +\frac{1}{2}\left[e^{i \hat{\varphi} / 2}\left(i t_{L} e^{i \varphi_{x} / 2} \hat{X}_{m} \hat{\sigma}_{d}+t_{R} \hat{Y}_{m} \hat{\sigma}_{d}\right)+\text { H.c. }\right] .
\end{aligned}
$$

We have performed a Jordan-Wigner transformation

$$
\begin{gathered}
\hat{d}=-\hat{\sigma}_{d}, \\
\hat{\gamma}_{2}=-\hat{Z}_{d} \hat{X}_{m}, \\
\hat{\gamma}_{3}=-\hat{Z}_{d} \hat{Y}_{m},
\end{gathered}
$$

where $\hat{\sigma}_{j}=|0\rangle_{j}\langle 1|$ is a two-level system lowering operator, and $\hat{X}_{j}=\hat{\sigma}_{j}^{\dagger}+\hat{\sigma}_{j} \hat{Y}_{j}=i\left(\hat{\sigma}_{j}^{\dagger}-\hat{\sigma}_{j}\right)$ and $\hat{Z}_{j}=\hat{\sigma}_{j} \hat{\sigma}_{j}^{\dagger}-$ $\hat{\sigma}_{j}^{\dagger} \hat{\sigma}_{j}$ are the usual Pauli matrices with $j=d, m$.

To diagonalize Eq. (D1), we follow Ref. [30]. We can label a basis for the system by $\left|N, n_{d}, a\right\rangle$, where $N \in \mathbb{Z}$, $n_{d}=0,1$, and $a= \pm$ correspond to the island charge, dot occupation number, and Majorana parity, respectively. Note that the total charge $\hat{N}+\hat{\sigma}_{d}^{\dagger} \hat{\sigma}_{d}$ is conserved by 
Eq. (D1). We therefore define projectors

$$
\begin{aligned}
\hat{P}_{n} & =\left|0_{n}\right\rangle\left\langle 0_{n}|+| 1_{n}\right\rangle\left\langle 1_{n}\right|, \\
\left|0_{n}\right\rangle & =\left|N=n, n_{d}=0\right\rangle, \\
\left|1_{n}\right\rangle & =\left|N=n-1, n_{d}=1\right\rangle,
\end{aligned}
$$

and use the fact that $\hat{H}_{\mathrm{MB}}=\sum_{n} \hat{H}_{\mathrm{MB}, n}$ with

$$
\hat{H}_{\mathrm{MB}, n}=\hat{P}_{n} \hat{H}_{\mathrm{MB}} \hat{P}_{n} .
$$

The Hamiltonian projected onto the $n$th subspace can be diagonalized by a unitary (see Ref. [30] for details)

$$
\hat{U}_{n}=e^{-\left[\alpha-(n) \hat{\sigma}_{n}^{\dagger} \hat{\sigma}_{m}-\alpha_{+}(n) \hat{\sigma}_{n}^{\dagger} \hat{\sigma}_{m}^{\dagger}-\text { H.c. }\right]}
$$

with

$$
\tan \left[2\left|\alpha_{ \pm}(n)\right|\right]=\frac{\left|t_{L} e^{i \phi_{x} / 2} \pm t_{R}\right|}{2 \delta(n)}
$$

and we have defined a lowering operator within the $n$th subspace

$$
\hat{\sigma}_{n}=\hat{P}_{n} e^{i \hat{\varphi} / 2} \hat{\sigma}_{d} \hat{P}_{n}=\left|0_{n}\right\rangle\left\langle 1_{n}\right| .
$$

Explicitly, we find that

$$
\begin{aligned}
\hat{H}_{\mathrm{MB}, n}^{\prime} & =\hat{U}_{n}^{\dagger} \hat{H}_{\mathrm{MB}, n} \hat{U}_{n} \\
& =\varepsilon_{c}(n) \hat{\sigma}_{n}^{\dagger} \hat{\sigma}_{n}+\varepsilon_{m}(n) \hat{\sigma}_{m}^{\dagger} \hat{\sigma}_{m}+E(n),
\end{aligned}
$$

where

$$
\begin{aligned}
\varepsilon_{c}(n) & =\frac{\operatorname{sgn} \delta(n)}{2}\left[f_{+}(n)+f_{-}(n)\right], \\
\varepsilon_{m}(n) & =\frac{\operatorname{sgn} \delta(n)}{2}\left[f_{+}(n)-f_{-}(n)\right], \\
E(n) & =E_{\mathrm{tot}}\left(n-n_{g}\right)^{2}+\frac{\delta(n)-\varepsilon_{c}(n)-\varepsilon_{m}(n)}{2}, \\
f_{ \pm}(n) & =\sqrt{\delta(n)^{2}+t_{L}^{2}+t_{R}^{2} \pm 2 t_{L} t_{R} \cos \left(\frac{\varphi_{x}}{2}\right)}, \\
\delta(n) & =E_{\mathrm{tot}}+\varepsilon-2 E_{\mathrm{tot}}\left(n-n_{g}\right) .
\end{aligned}
$$

To recover the full Hamiltonian given in Eq. (20), we use $\hat{H}_{\mathrm{MB}}^{\prime}=\sum_{n} \hat{H}_{\mathrm{MB}, n}^{\prime} \hat{P}_{n}$ and reverse the Jordan-Wigner transformation, Eq. (D2).

The qubit-resonator interaction Hamiltonian $\hat{H}_{\text {int }}$, defined in Eq. (25), also preserves the total charge and can similarly be written as $\hat{H}_{\mathrm{int}}=\sum_{n} \hat{H}_{\mathrm{int}, n} \hat{P}_{n}$ with

$$
\hat{H}_{\mathrm{int}, n}=i \hbar \lambda\left(n-\hat{\sigma}_{n}^{\dagger} \hat{\sigma}_{n}\right)\left(\hat{a}^{\dagger}-\hat{a}\right) \text {. }
$$

Following Ref. [30], we perform the same unitary transformation as in Eq. (D8) to find that

$$
\hat{H}_{\mathrm{int}, n}^{\prime}=\hat{U}_{n}^{\dagger} \hat{H}_{\mathrm{int}, n} \hat{U}_{n}=i \hbar \lambda \hat{N}_{n}^{\prime}\left(\hat{a}^{\dagger}-\hat{a}\right),
$$

where

$$
\begin{aligned}
\lambda \hat{N}_{n}^{\prime}= & \lambda n+g_{c}(n) \hat{\sigma}_{n}^{\dagger} \hat{\sigma}_{n}+g_{m}(n) \hat{\sigma}_{m}^{\dagger} \hat{\sigma}_{m} \\
& +\left[g_{+}(n) \hat{\sigma}_{m} \hat{\sigma}_{n}+g_{-}(n) \hat{\sigma}_{m}^{\dagger} \hat{\sigma}_{n}+\text { H.c. }\right]
\end{aligned}
$$

and

$$
\begin{aligned}
& g_{c}(n)=-\frac{\lambda}{2}\left(\frac{\delta(n)}{f_{+}(n)}+\frac{\delta(n)}{f_{-}(n)}\right), \\
& g_{m}(n)=-\frac{\lambda}{2}\left(\frac{\delta(n)}{f_{+}(n)}-\frac{\delta(n)}{f_{-}(n)}\right), \\
& g_{ \pm}(n)=-\frac{\lambda}{2} \frac{i\left(t_{L} e^{i \varphi_{x} / 2} \pm t_{R}\right)}{f_{ \pm}(n)} .
\end{aligned}
$$

The result in Eq. (22) is found by setting $n_{g}=0, t_{L, R}=t$, projecting onto the $n=0$ total charge sector, and reversing the Jordan-Wigner transformation.

\section{Calculating the dispersive shift}

We can now calculate the dispersive shift from the complete qubit-resonator Hamiltonian in the $n$th subspace

$$
\hat{H}_{n}^{\prime}=\hat{H}_{r}+\hat{H}_{\mathrm{MB}, n}^{\prime}+\hat{H}_{\mathrm{in}, n}^{\prime} .
$$

The eigenstates of $\hat{H}_{\mathrm{MB}, n}^{\prime}$ for each subspace $n$ can be labeled $\left|n-\sigma_{n}, \sigma_{n}, a\right\rangle$ with $\sigma_{n}=0,1$ the occupancy of $\hat{\sigma}_{n}^{\dagger} \hat{\sigma}_{n}$ and $a= \pm$ corresponding to the Majorana parity $2 \hat{\sigma}_{m}^{\dagger} \hat{\sigma}_{m}-1$. We proceed by calculating the dispersive frequency shift of the resonator

$$
\chi_{m b, n}=\frac{1}{2}\left(\chi_{n, 0,+}-\chi_{n, 0,-}\right)
$$

for the two states $|1\rangle=|n, 0,+\rangle$ and $|0\rangle=|n, 0,-\rangle$, where we assume that the dot is unoccupied for the two qubit states. From Eq. (D12) we have the following nonzero, off-diagonal matrix elements:

$$
\begin{aligned}
\left|i \lambda\left\langle n, 0,-\left|\hat{N}_{n}^{\prime}\right| n-1,1,+\right\rangle\right| & =\left|g_{+}(n)\right|, \\
\left|i \lambda\left\langle n, 0,+\left|\hat{N}_{n}^{\prime}\right| n-1,1,-\right\rangle\right| & =\left|g_{-}(n)\right| .
\end{aligned}
$$

The diagonal matrix elements, corresponding to the first three terms in Eq. (D12), cancel out when calculating the dispersive shifts by Eq. (A3a). 
In the logical qubit subspace we have two transitions: $|n, 0,+\rangle \leftrightarrow|n-1,1,-\rangle$ with energy $\varepsilon_{c}(n)-\varepsilon_{m}(n)=$ $f_{-}(n)$ and $|n, 0,-\rangle \leftrightarrow|n-1,1,+\rangle$ with energy $\varepsilon_{c}(n)+$ $\varepsilon_{m}(n)=f_{+}(n)$. From Eq. (A3a), we obtain

$$
\begin{aligned}
\chi_{n, 0,+}= & \frac{\left|i \lambda\left\langle n, 0,+\left|\hat{N}_{n}^{\prime}\right| n-1,1,-\right\rangle\right|^{2}}{-f_{-}(n) / \hbar-\omega_{r}} \\
& -\frac{\left|i \lambda\left\langle n-1,1,-\left|\hat{N}_{n}^{\prime}\right| n, 0,+\right\rangle\right|^{2}}{f_{-}(n) / \hbar-\omega_{r}} \\
= & -\frac{\left|g_{-}(n)\right|^{2}}{f_{-}(n) / \hbar+\omega_{r}}-\frac{\left|g_{-}(n)\right|^{2}}{f_{-}(n) / \hbar-\omega_{r}}
\end{aligned}
$$

and

$$
\begin{aligned}
\chi_{n, 0,-}= & \frac{\left|i \lambda\left\langle n, 0,-\left|\hat{N}_{n}^{\prime}\right| n-1,1,+\right\rangle\right|^{2}}{-f_{+}(n) / \hbar-\omega_{r}} \\
& -\frac{\left|i \lambda\left\langle n-1,1,+\left|\hat{N}_{n}^{\prime}\right| n, 0,-\right\rangle\right|^{2}}{f_{+}(n) / \hbar-\omega_{r}} \\
= & -\frac{\left|g_{+}(n)\right|^{2}}{f_{+}(n) / \hbar+\omega_{r}}-\frac{\left|g_{+}(n)\right|^{2}}{f_{+}(n) / \hbar-\omega_{r}} .
\end{aligned}
$$

Finally, from Eq. (D15) we have

$$
\chi_{m b, n} \simeq \frac{1}{2}\left(\frac{\left|g_{+}(n)\right|^{2}}{f_{+}(n) / \hbar-\omega_{r}}-\frac{\left|g_{-}(n)\right|^{2}}{f_{-}(n) / \hbar-\omega_{r}}\right)
$$

where we have again dropped fast-rotating terms approximately $1 /\left[f_{ \pm}(n) / \hbar+\omega_{r}\right]$ for simplicity. The result quoted in Eq. (28) corresponds to setting $n=0, n_{g}=0$, and $t_{L, R}=$ $t$.

\section{APPENDIX E: LONGITUDINAL READOUT FOR MAJORANA BOX QUBITS}

As alluded to in Sec. II, the parity protection of the Majorana box qubit dispersive readout scheme is not as strong as that of the Majorana transmon. This is apparent from the last two lines of Eq. (26), since the qubit-resonator interaction does not commute with the Majorana parity operator $i \hat{\gamma}_{2} \hat{\gamma}_{3}$ in the diagonal frame of $\hat{H}_{\mathrm{MB}}^{\prime}$. Approximate parity protection is recovered in the dispersive regime where Eq. (2) is valid.

An alternative approach was proposed in Ref. [30] that instead relies on exploiting the longitudinal Majoranaresonator interaction apparent in the first line of Eq. (26), quantified by $g_{m}$. Longitudinal coupling refers to an interaction that is diagonal in the qubit basis, in contrast to the off-diagonal, or transversal, coupling usually exploited for charge qubits. The readout scheme introduced in Ref. [30] uses a parametric modulation of the longitudinal coupling $g_{m}$ in Eq. (26). In practice, this can be achieved in a number of ways, including flux modulation and modulating the tunnel coupling $t$ and/or the detuning $\delta$.
The first line of Eq. (26) clearly commutes with $i \hat{\gamma}_{2} \hat{\gamma}_{3}$, and a QND readout protocol can therefore be achieved given that the parity nonconserving processes in the two last lines are heavily suppressed. This latter requirement can be met by placing the resonator sufficiently far offresonance from the relevant island-dot transitions. More precisely, for ideal longitudinal readout, the denominators $\left|f_{ \pm} / \hbar-\omega_{r}\right|$ in Eq. (28) should be sufficiently large such that $\chi_{m b}$ is negligible. This should be relatively straight forward to achieve, e.g., by increasing the charging and dot energies, $E_{\text {tot }}$ and $\varepsilon$. We note that increasing these energy scales might furthermore be beneficial from the point of view of quasiparticle poisoning $[25,26]$.

The infidelity of a longitudinal readout scheme for the Majorana box qubit is shown in Fig. 6(b). When producing these results, we take the coupling strength to be modulated at the resonator frequency, $g_{m}(t)=\bar{g}_{m}+\tilde{g}_{m} \cos \left(\omega_{r} t\right)$, and assume an ideal longitudinal interaction, which in a frame rotating at $\omega_{r}$ can be written as

$$
\hat{H}_{z}=\frac{i \tilde{g}_{z}}{2} i \hat{\gamma}_{2} \hat{\gamma}_{3}\left(\hat{a}^{\dagger}-\hat{a}\right)
$$

where $\tilde{g}_{z}=\tilde{g}_{m} / 2$ is the longitudinal modulation and fast rotating terms are neglected.

We refer the reader to Refs. [30,57] for further details. Here the fidelity is calculated using Eq. (30) with the following expression for the SNR [57]:

$$
\mathrm{SNR}_{l}=\sqrt{8}\left|\tilde{g}_{z}\right| \sqrt{\frac{\tau}{\kappa}}\left[1-\frac{2}{\kappa \tau}\left(1-e^{-\kappa \tau / 2}\right)\right] .
$$

For the sake of comparison in Fig. 6, we set $\kappa$ and the intracavity photon number $\bar{n}=\left(\tilde{g}_{z} / \kappa\right)^{2}$ [57] to be the same for the dispersive and longitudinal readout protocols. For the parameters in the figure, this corresponds to a modulation of $\tilde{g}_{z} / 2 \pi \simeq 10 \mathrm{MHz}$. For concreteness, we illustrate the
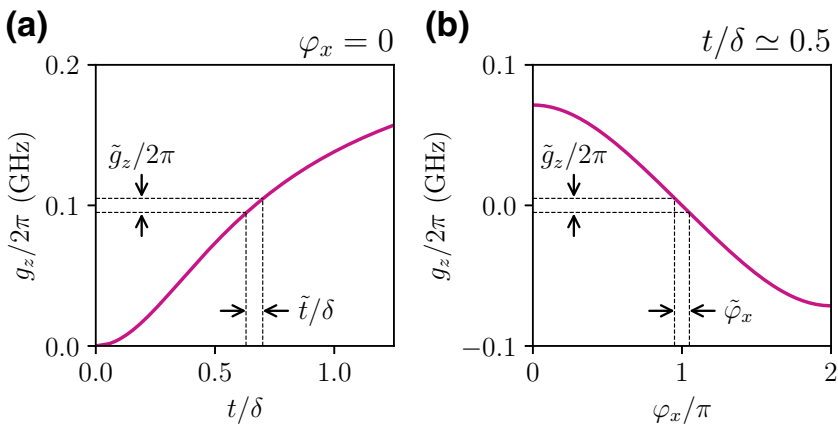

FIG. 8. Longitudinal coupling strength for the Majorana box qubit $g_{z}=g_{m} / 2$ as a function of $t / \delta$ and $\varphi_{x}$. For (a), we have set $\varphi_{x}=0$ and, for (b), we have set $t / \delta \simeq 0.5$. Dashed lines indicate a modulation of $\tilde{g}_{z}=10 \mathrm{MHz}$, which was used in Fig. 6. This corresponds to a modulation of roughly (a) $\tilde{t} / \delta \simeq 0.1$ or (b) $\tilde{\varphi}_{x}=$ $\pi / 10$. 
range of modulation of $t / \delta$ and $\varphi_{x}$, respectively, needed to achieve $\tilde{g}_{z} / 2 \pi=10 \mathrm{MHz}$ in Fig. 8 for an example parameter set. These results clearly show that even a modest modulation in external parameters can lead to extremely fast longitudinal readout.

\section{ACKNOWLEDGMENTS}

We thank Andrew Doherty and Torsten Karzig for useful discussions. This research is supported by the Australian Research Council, through the Centre of Excellence for Engineered Quantum Systems (EQUS) under Grant No. CE170100009 and the Discovery Early Career Research Award under Grant No. DE190100380.

[1] A. Y. Kitaev, Fault-tolerant quantum computation by anyons, Ann. Phys. 303, 2 (2003).

[2] S. Bravyi, Universal quantum computation with the $v=$ 5/2 fractional quantum hall state, Phys. Rev. A 73, 042313 (2006).

[3] C. Nayak, S. H. Simon, A. Stern, M. Freedman, and S. Das Sarma, Non-Abelian anyons and topological quantum computation, Rev. Mod. Phys. 80, 1083 (2008).

[4] A. Y. Kitaev, Unpaired Majorana fermions in quantum wires, Phys.-Usp. 44, 131 (2001).

[5] L. Fu and C. L. Kane, Superconducting Proximity Effect and Majorana Fermions at the Surface of a Topological Insulator, Phys. Rev. Lett. 100, 096407 (2008).

[6] R. M. Lutchyn, J. D. Sau, and S. Das Sarma, Majorana Fermions and a Topological Phase Transition in Semiconductor-Superconductor Heterostructures, Phys. Rev. Lett. 105, 077001 (2010).

[7] Y. Oreg, G. Refael, and F. von Oppen, Helical Liquids and Majorana Bound States in Quantum Wires, Phys. Rev. Lett. 105, 177002 (2010).

[8] J. D. Sau, R. M. Lutchyn, S. Tewari, and S. Das Sarma, Generic New Platform for Topological Quantum Computation Using Semiconductor Heterostructures, Phys. Rev. Lett. 104, 040502 (2010).

[9] J. Alicea, New directions in the pursuit of Majorana fermions in solid state systems, Rep. Prog. Phys. 75, 076501 (2012).

[10] V. Mourik, K. Zuo, S. M. Frolov, S. R. Plissard, E. P. A. M. Bakkers, and L. P. Kouwenhoven, Signatures of Majorana fermions in hybrid superconductor-semiconductor nanowire devices, Science 336, 1003 (2012).

[11] C. W. J. Beenakker, Search for Majorana fermions in superconductors, Annu. Rev. Condens. Matter Phys. 4, 113 (2013).

[12] S. Nadj-Perge, I. K. Drozdov, J. Li, H. Chen, S. Jeon, J. Seo, A. H. MacDonald, B. A. Bernevig, and A. Yazdani, Observation of Majorana fermions in ferromagnetic atomic chains on a superconductor, Science 346, 602 (2014).

[13] S. M. Albrecht, A. P. Higginbotham, M. Madsen, F. Kuemmeth, T. S. Jespersen, J. Nygård, P. Krogstrup, and C. M. Marcus, Exponential protection of zero modes in Majorana islands, Nature 531, 206 (2016).
[14] M. T. Deng, S. Vaitiekènas, E. B. Hansen, J. Danon, M. Leijnse, K. Flensberg, J. Nygård, P. Krogstrup, and C. M. Marcus, Majorana bound state in a coupled quantum-dot hybrid-nanowire system, Science 354, 1557 (2016).

[15] F. Nichele, A. C. C. Drachmann, A. M. Whiticar, E. C. T. O'Farrell, H. J. Suominen, A. Fornieri, T. Wang, G. C. Gardner, C. Thomas, A. T. Hatke, P. Krogstrup, M. J. Manfra, K. Flensberg, and C. M. Marcus, Scaling of Majorana Zero-Bias Conductance Peaks, Phys. Rev. Lett. 119, 136803 (2017).

[16] R. M. Lutchyn, E. P. A. M. Bakkers, L. P. Kouwenhoven, P. Krogstrup, C. M. Marcus, and Y. Oreg, Majorana zero modes in superconductorsemiconductor heterostructures, Nat. Rev. Mater. 3, 52 (2018).

[17] M. Leijnse and K. Flensberg, Introduction to topological superconductivity and Majorana fermions, Semicond. Sci. Technol. 27, 124003 (2012).

[18] T. D. Stanescu and S. Tewari, Majorana fermions in semiconductor nanowires: fundamentals, modeling, and experiment, J. Phys. Condens. Matter 25, 233201 (2013).

[19] J. Alicea, Y. Oreg, G. Refael, F. von Oppen, and M. P. A. Fisher, Non-Abelian statistics and topological quantum information processing in 1D wire networks, Nat. Phys. 7, 412 (2011).

[20] J. D. Sau, D. J. Clarke, and S. Tewari, Controlling nonabelian statistics of Majorana fermions in semiconductor nanowires, Phys. Rev. B 84, 094505 (2011).

[21] B. van Heck, A. R. Akhmerov, F. Hassler, M. Burrello, and C. W. J. Beenakker, Coulomb-assisted braiding of Majorana fermions in a Josephson junction array, New J. Phys. 14, 035019 (2012).

[22] T. Hyart, B. van Heck, I. C. Fulga, M. Burrello, A. R. Akhmerov, and C. W. J. Beenakker, Flux-controlled quantum computation with Majorana fermions, Phys. Rev. B 88, 035121 (2013)

[23] S. Das Sarma, M. Freedman, and C. Nayak, Majorana zero modes and topological quantum computation, npj Quantum Inf. 1, 15001 (2015).

[24] D. Aasen, M. Hell, R. V. Mishmash, A. Higginbotham, J. Danon, M. Leijnse, T. S. Jespersen, J. A. Folk, C. M. Marcus, K. Flensberg, and J. Alicea, Milestones Toward Majorana-Based Quantum Computing, Phys. Rev. X 6, 031016 (2016)

[25] S. Plugge, A. Rasmussen, R. Egger, and K. Flensberg, Majorana box qubits, New J. Phys. 19, 012001 (2017).

[26] T. Karzig, C. Knapp, R. M. Lutchyn, P. Bonderson, M. B. Hastings, C. Nayak, J. Alicea, K. Flensberg, S. Plugge, Y. Oreg, C. M. Marcus, and M. H. Freedman, Scalable designs for quasiparticle-poisoning-protected topological quantum computation with Majorana zero modes, Phys. Rev. B 95, 235305 (2017).

[27] C. Ohm and F. Hassler, Microwave readout of Majorana qubits, Phys. Rev. B 91, 085406 (2015).

[28] K. Gharavi, D. Hoving, and J. Baugh, Readout of Majorana parity states using a quantum dot, Phys. Rev. B 94, 155417 (2016).

[29] T. Li, W. A. Coish, M. Hell, K. Flensberg, and M. Leijnse, Four-Majorana qubit with charge readout: Dynamics and decoherence, Phys. Rev. B 98, 205403 (2018). 
[30] A. L. Grimsmo and T. B. Smith, Majorana qubit readout using longitudinal qubit-resonator interaction, Phys. Rev. B 99, 235420 (2019).

[31] G. Széchenyi and A. Pályi, Parity-to-charge conversion for readout of topological Majorana qubits, Phys. Rev. B 101, 235441 (2020).

[32] J. F. Steiner and F. von Oppen, Readout of Majorana qubits, Phys. Rev. Res. 2, 033255 (2020).

[33] M. I. K. Munk, J. Schulenborg, R. Egger, and K. Flensberg, Parity-to-charge conversion in Majorana qubit readout, Phys. Rev. Res. 2, 033254 (2020).

[34] V. D. Maman, M. Gonzalez-Zalba, and A. Pályi, Charge noise and overdrive errors in reflectometry-based charge, spin and majorana qubit readout, arXiv:2006.12391 [condmat.mes-hall] (2020).

[35] A. Khindanov, D. Pikulin, and T. Karzig, Visibility of noisy quantum dot-based measurements of Majorana qubits, arXiv:2007.11024 [cond-mat.mes-hall] (2020).

[36] P. Bonderson, M. Freedman, and C. Nayak, MeasurementOnly Topological Quantum Computation, Phys. Rev. Lett. 101, 010501 (2008).

[37] P. Bonderson, M. Freedman, and C. Nayak, Measurementonly topological quantum computation via anyonic interferometry, Ann. Phys. 324, 787 (2009).

[38] T. Karzig, Y. Oreg, G. Refael, and M. H. Freedman, Robust Majorana magic gates via measurements, Phys. Rev. B 99, 144521 (2019).

[39] A. Blais, A. L. Grimsmo, S. M. Girvin, and A. Wallraff, Circuit Quantum Electrodynamics, arXiv:2005.12667 [quant-ph] (2020).

[40] A. Wallraff, D. I. Schuster, A. Blais, L. Frunzio, R.S. Huang, J. Majer, S. Kumar, S. M. Girvin, and R. J. Schoelkopf, Strong coupling of a single photon to a superconducting qubit using circuit quantum electrodynamics, Nature 431, 162 (2004).

[41] T. Walter, P. Kurpiers, S. Gasparinetti, P. Magnard, A. Potočnik, Y. Salathé, M. Pechal, M. Mondal, M. Oppliger, C. Eichler, and A. Wallraff, Rapid HighFidelity Single-Shot Dispersive Readout of Superconducting Qubits, Phys. Rev. Appl. 7, 054020 (2017).

[42] J. I. Colless, A. C. Mahoney, J. M. Hornibrook, A. C. Doherty, H. Lu, A. C. Gossard, and D. J. Reilly, Dispersive Readout of a Few-Electron Double Quantum Dot with Fast rf Gate Sensors, Phys. Rev. Lett. 110, 046805 (2013).

[43] A. West, B. Hensen, A. Jouan, T. Tanttu, C.-H. Yang, A. Rossi, M. F. Gonzalez-Zalba, F. Hudson, A. Morello, D. J. Reilly, and A. S. Dzurak, Gate-based single-shot readout of spins in silicon, Nat. Nanotechnol. 14, 437 (2019).

[44] D. de Jong, J. van Veen, L. Binci, A. Singh, P. Krogstrup, L. P. Kouwenhoven, W. Pfaff, and J. D. Watson, Rapid Detection of Coherent Tunneling in an InAs Nanowire Quantum Dot through Dispersive Gate Sensing, Phys. Rev. Appl. 11, 044061 (2019).

[45] T. W. Larsen, K. D. Petersson, F. Kuemmeth, T. S. Jespersen, P. Krogstrup, J. Nygård, and C. M. Marcus, Semiconductor-Nanowire-Based Superconducting Qubit, Phys. Rev. Lett. 115, 127001 (2015).
[46] G. de Lange, B. van Heck, A. Bruno, D. J. van Woerkom, A. Geresdi, S. R. Plissard, E. P. A. M. Bakkers, A. R. Akhmerov, and L. DiCarlo, Realization of Microwave Quantum Circuits Using Hybrid Superconducting-Semiconducting Nanowire Josephson Elements, Phys. Rev. Lett. 115, 127002 (2015).

[47] E. Ginossar and E. Grosfeld, Microwave transitions as a signature of coherent parity mixing effects in the Majoranatransmon qubit, Nat. Commun. 5, 1 (2014).

[48] K. Yavilberg, E. Ginossar, and E. Grosfeld, Fermion parity measurement and control in Majorana circuit quantum electrodynamics, Phys. Rev. B 92, 075143 (2015).

[49] M. Hell, J. Danon, K. Flensberg, and M. Leijnse, Time scales for Majorana manipulation using Coulomb blockade in gate-controlled superconducting nanowires, Phys. Rev. B 94, 035424 (2016).

[50] A. Blais, R.-S. Huang, A. Wallraff, S. M. Girvin, and R. J. Schoelkopf, Cavity quantum electrodynamics for superconducting electrical circuits: An architecture for quantum computation, Phys. Rev. A 69, 062320 (2004).

[51] J. Koch, T. M. Yu, J. Gambetta, A. A. Houck, D. I. Schuster, J. Majer, A. Blais, M. H. Devoret, S. M. Girvin, and R. J. Schoelkopf, Charge-insensitive qubit design derived from the Cooper pair box, Phys. Rev. A 76, 042319 (2007).

[52] C. Knapp, T. Karzig, R. M. Lutchyn, and C. Nayak, Dephasing of Majorana-based qubits, Phys. Rev. B 97, 125404 (2018).

[53] T. Karzig, W. S. Cole, and D. I. Pikulin, Quasiparticle poisoning of Majorana qubits, arXiv:2004.01264 [condmat.mes-hall] (2020).

[54] M.-T. Deng, S. Vaitiekènas, E. Prada, P. San-Jose, J. Nygård, P. Krogstrup, R. Aguado, and C. M. Marcus, Nonlocality of Majorana modes in hybrid nanowires, Phys. Rev. B 98, 085125 (2018).

[55] A. A. Houck, J. A. Schreier, B. R. Johnson, J. M. Chow, J. Koch, J. M. Gambetta, D. I. Schuster, L. Frunzio, M. H. Devoret, S. M. Girvin, and R. J. Schoelkopf, Controlling the Spontaneous Emission of a Superconducting Transmon Qubit, Phys. Rev. Lett. 101, 080502 (2008).

[56] L. Fu, Electron Teleportation via Majorana Bound States in a Mesoscopic Superconductor, Phys. Rev. Lett. 104, 056402 (2010).

[57] N. Didier, J. Bourassa, and A. Blais, Fast Quantum Nondemolition Readout by Parametric Modulation of Longitudinal Qubit-Oscillator Interaction, Phys. Rev. Lett. 115, 203601 (2015).

[58] G. Zhu, D. G. Ferguson, V. E. Manucharyan, and J. Koch, Circuit QED with fluxonium qubits: Theory of the dispersive regime, Phys. Rev. B 87, 024510 (2013).

[59] S. Bravyi, D. P. DiVincenzo, and D. Loss, SchriefferWolff transformation for quantum many-body systems, Ann. Phys. 326, 2793 (2011).

[60] R. Winkler, Spin-Orbit coupling effects in two-dimensional electron and hole systems, Springer Tracts Mod. Phys. 191 (2003). 